\title{
A geometric-sensitivity-difference based algorithm improves object depth-localization for diffuse optical tomography in a circular-array outward-imaging geometry
}

\author{
Guan Xu \\ Department of Radiology, Medical School, University of Michigan, Ann Arbor, Michigan 48109 \\ Daqing Piao ${ }^{\text {a) }}$ \\ School of Electrical and Computer Engineering, Oklahoma State University, Stillwater, Oklahoma 74078
}

(Received 11 June 2012; revised 8 November 2012; accepted for publication 27 November 2012; published 27 December 2012)

\begin{abstract}
Purpose: To improve object depth-localization for diffuse optical tomography (DOT) in a circulararray outward-imaging geometry that is subjected to strong sensitivity variation with respect to imaging depth.

Methods: The authors introduce an alternative DOT image reconstruction approach that optimizes the data-model fit based on the paired measurements corresponding to two pairs of source-detector that share either the source or the detector, in comparison to the conventional method that optimizes the data-model fit based on the unpaired measurements corresponding to individual pairs of source-detector. This alternative approach, namely, geometric-sensitivity-difference (GSD) method, effectively reduces the variation of the reconstruction sensitivity with respect to imaging depth. The DOT image reconstruction based on GSD-scheme applied to same-source source-detector pairs is demonstrated using simulated and experimental continuous-wave measurements in a circular-array outward-imaging geometry, of which the native sensitivity varies strongly with respect to the depth. The outcomes of GSD-based image reconstruction are compared to those of two other methods: one is the conventional baseline method that utilizes the native sensitivity but does not involve depthcompensating scheme; and the other is a reference-compensation approach that employs active and depth-adapted compensation scheme to counteract the dependence of the reconstruction sensitivity with respect to imaging depth.
\end{abstract}

Results: The GSD method generally outperforms the other two methods in localizing the depth of single object, resolving two objects that are azimuthally separated, and estimating the optical property of single object or azimuthally separated dual objects. The GSD method, however, demands more computations due to an increase of the element size of the resulted sensitivity matrix and more matrix multiplications.

Conclusions: The GSD method improves the depth localization in the circular-array outwardimaging geometry, by taking advantage of the paired measurements of two source-sharing source-detector-pairs to passively and effectively homogenize the sensitivity of the reconstruction with respect to imaging depth. (c) 2013 American Association of Physicists in Medicine. [http://dx.doi.org/10.1118/1.4771957]

Key words: image reconstruction, diffuse optical tomography, sensitivity, photon migration in tissue

\section{INTRODUCTION}

Diffuse optical tomography (DOT) is inherently prone to spatially dependent sensitivity, ${ }^{1-4}$ due to scattering-dominated photon propagation in biological tissue. ${ }^{5}$ The spatial dependence of DOT sensitivity is also specific to the geometry of the interface between the medium and the array of optodes. The optode-array of DOT usually has one- or two-dimensional symmetry ${ }^{2,6-9}$ that gives rise to a sensitivity distribution that is mostly uniform along the direction of the symmetry except at locations close to the optodes. For example, the spatial sensitivity of a circular array ${ }^{8,9}$ that has evenly distributed optodes along the circumference is azimuthally invariant, and the spatial sensitivity of a near-planar array whereupon the optodes are orderly distributed ${ }^{3,7}$ changes insignificantly over the lateral dimension of the array. However, at the directions orthogonal to the symmetry of the optode configuration, specifically along the depth into the medium, the spatial sensitivity varies, generally, with a pronounced peak in the proximity of the medium-array interface, as exemplified by the thinner solid curve in Fig. 1(a) (detailed description of Fig. 1 is referred to Sec. II.A). Such variation of the sensitivity causes depth-dependent reconstruction of the contrast and resolution. More severely, it may cause objects of different depths to be recovered at approximately the same depth, the position at which the radial profile of the sensitivity peaks. ${ }^{1-3,7,8}$ The depth-dependence of the sensitivity is thus a common issue to be negotiated in many DOT applications, including those to brain, ${ }^{7,10}$ to breast using planar remission geometry, ${ }^{1}$ and to prostate via endorectal probing for either sagittal $^{3}$ or axial ${ }^{9}$ imaging, etc. Among these DOT applications, the endorectal axial-imaging geometry is subjected to 


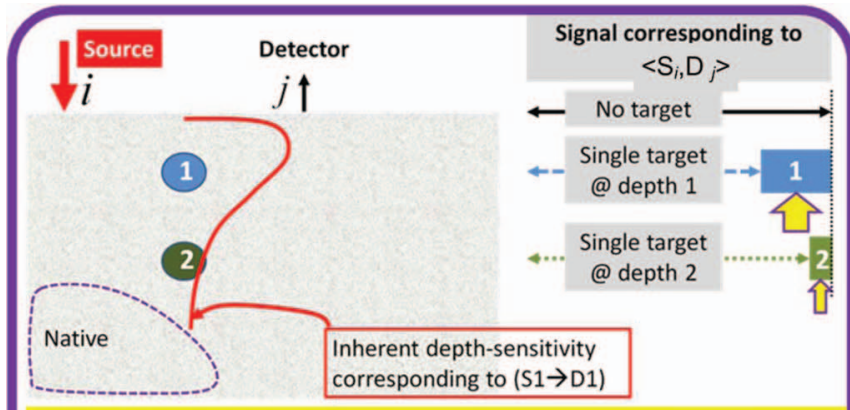

(a) Native sensitivity

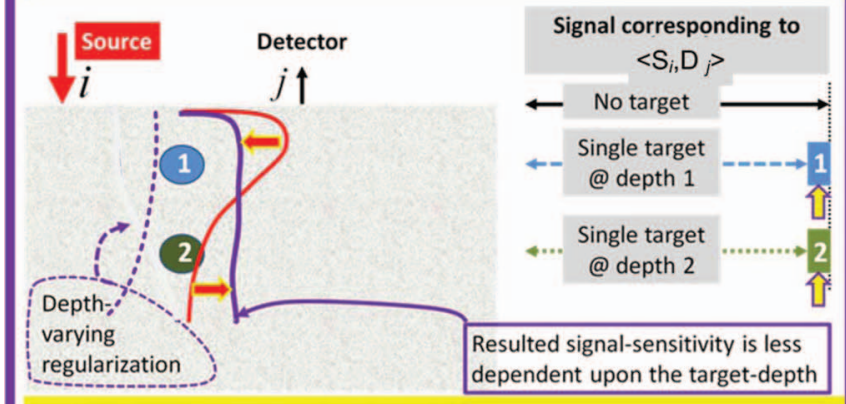

(b) Varying regularization

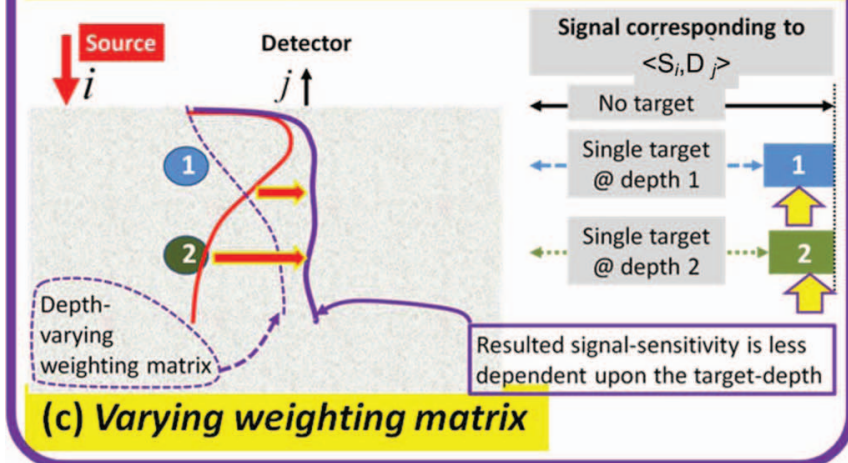

FIG. 1. Illustration of conventional DOT image reconstruction approaches that utilize the measurements of individual source-detector-pairs. The thinner solid curve represents the native sensitivity profile, the thicker solid curve represents the modified sensitivity profile, and the dashed curve indicates the regularization or compensation with respect to imaging depth. The boxes pointed by framed arrows represent the signal responses to an object at position 1 and 2, respectively. (a) The signal responses to an object located at different depths are different due to the spatial variations of the native sensitivity. (b) Spatially varying regularization factors suppresses the stronger intensity of the natural sensitivity during the sensitivity matrix inversion to even the recovery of the object contrast with respect to imaging depth. (c) Spatially varying weighting magnifies the weak sensitivity in deeper regions and to even the recovery of the object contrast with respect to imaging depth.

arguably the strongest variation of the sensitivity with respect to imaging depth, due to the rapid reduction of photon fluence versus the source-detector distance when compared with the geometries involving a planar interface or a curved interface that encloses the medium. ${ }^{11}$ As will be shown, localizing the depth of an object of interest in such circular-array outward-imaging geometry is challenging.

Robust localization of an object in the depth direction along which the sensitivity varies significantly has to rely upon establishing a reconstruction scheme in which the up- dating of the medium optical properties is insensitive to the imaging depth. Methods demonstrated to suppress the sensitivity of reconstruction to the imaging depth can be coarsely classified into two categories. The first category, as conceptually illustrated in Fig. 1(b), applies stronger regularization to regions with higher native sensitivity to damp the response of the reconstruction to the same amount of the update to the optical properties. For a circular-array inward-imaging geometry of which the spatial sensitivity tends to be azimuthally uniform but decreases toward the center of the imaging geometry, Pogue et al. applied a radially varying regularization parameter that had a greater magnitude near the sources but smaller magnitude in the interior of the geometry. ${ }^{8}$ This method, called spatially variant regularization (SVR), noticeably improved the reconstruction, as the artifacts near the optode array were suppressed and the contrast and resolution across the image were equalized. However, artifacts could appear at the center of the circular image, and the parameters determining the radial variation of the regularization were required to be optimized for a particular imaging geometry and a specific object size. It is also noted that the spatial sensitivity of the circular-array inward-imaging geometry utilized by Pogue et $a l .{ }^{8}$ decreases only mildly versus the depth from the medium-boundary, when compared to that of the circular-array outward-imaging geometry to be investigated in this study. The second category of methods, as conceptually illustrated in Fig. 1(c), implements a spatially resolved weighting scheme to the native depth-dependent sensitivity in the iteration process. For a near-planar geometry of which the variation of the spatial sensitivity with respect to depth was stronger than that of the inward-imaging geometries of Ref. 8, Culver et al. employed spatially varied contrast-to-noise-ratio weighting to the reconstruction, after the data were corrected for optode mispositioning, to improve both the point-spread-function and the object-localization in depth. ${ }^{10}$ For geometries that were similar to the near-planar one in Ref. 10, Niu et al., ${ }^{2}$ Huang and Zhu, ${ }^{1}$ and Zhao et al. ${ }^{4}$ embedded depth-dependent weighting matrices in the iterative process to counterbalance the depth-variations of the respective native spatial sensitivities. The methods ${ }^{1,2,4}$ of the second category effectively elevated the response of the objective functions to the same amount of the update to the optical properties in deeper depths; however, the spatially varied weighting to the sensitivity profiles, such as that introduced by the depth-compensation-algorithm (DCA), ${ }^{2}$ naturally has to be optimized case-by-case.

These previously demonstrated methods, all making the reconstruction less sensitive to the object depth, may be different in terms of how the native sensitivity profile was compensated or counteracted. As in some the actions were imposed indirectly by the regularization but in others were applied directly to the sensitivity function; however, all these methods were similar, in terms of fitting the calculated data to the individual measurements taken by individual source-detectorpairs. This study introduces an alternative method of optimizing the data-model fit, which is to fit the calculated data to the paired measurements taken by two source-detectorpairs that share either the source or the detector. An intuitive 
explanation of this method is that, although the sensitivity of one pair of source-detector with respect to an object could vary significantly over the depth of the object, the relative response between two pairs of source-detector that share one optode could vary substantially less over the depth with respect to the same object, and hypothetically the image reconstruction based on this relative or paired response could be more robust in localizing the object than that based on the response of individual pairs of source-detector. The relative response between two optode-sharing source-detector-pairs to a given medium heterogeneity is related to the positioning (i.e., geometric) difference of the heterogeneity with respect to the two source-detector-pairs, therefore, the reconstruction scheme that takes advantage of such relative or paired response is called a geometric-sensitivity-difference (GSD) based reconstruction. The objective of this study is to demonstrate that the GSD method effectively evens the reconstruction updating sensitivity with respect to imaging depth and consequently improves DOT depth-localization. The GSD differs from previously investigated depth-compensation reconstruction method in that it does not involve depth-adapted or to-be-optimized parameters in order to reduce the variation of the reconstruction sensitivity with respect to imaging depth.

The rest of the paper is arranged as follows. Section II presents the analytical formations of GSD method as it applies to DOT image reconstruction in the context of Levenberg-Marquardt (LM) minimization, ${ }^{12}$ using paired continuous-wave $(\mathrm{CW})$ measurements from source-sharing source-detector-pairs. Section III outlines the circular-array axial outward-imaging geometry to be studied, of which the native sensitivity with respect to imaging depth varies significantly more than those of planar or circular-array inwardimaging geometries of previous studies. Section III also describes two reconstruction approaches against which the GSD method will be evaluated: one is a conventional or baseline method that applies a spatially invariant regulation in the LM minimization; ${ }^{13}$ and the other is a reference-compensation approach which is similar in methodology to the DCA method but is more robust than the original DCA method for the circular-array outward-imaging geometry of this study. Section IV details simulation and experimental methods used for evaluating the three reconstruction methods. The simulation and experimental results in Sec. V demonstrate that the GSD method generally outperforms the baseline and reference-compensation methods, in terms of localizing the depth of single object, resolving two azimuthally separated objects, and estimating the optical property of single object or azimuthally separated dual objects in the circular-array outward-imaging geometry. As all three methods involve a step-specific regularization scheme in the iterations, the same step-specific regularization factor optimal to the referencecompensation method is applied to the baseline reconstruction and the GSD based reconstruction. A step-specific regularization factor optimal to the baseline method for the studied geometry was found difficult to determine, because the baseline method performs very poorly in the studied geometry, as to impair the evaluation of the outcomes at different choices of the step-specific regularization factors. The step-specific regularization factor optimal to the reference-compensation method is not necessarily optimal to the GSD method. An outperformance of the GSD method over the baseline method is unsurprising at all, because the effective sensitivity profile of the former method is much more uniform with respect to the imaging depth than that of the latter method. The outperformance of the GSD method over the reference-compensation method shall relate to the pairing measurements by the former method versus the unpaired measurements by the latter method. Section VI discusses how the GSD operation may be implemented to Tikhonov minimization and the general-leastsquare (GLS) minimization. ${ }^{13}$

\section{THE GEOMETRIC-SENSITIVITY-DIFFERENCE METHOD}

The model of frequency-domain (FD) photon propagation in turbid media by using a diffusion approximation to the Boltzmann transport equation results in the following Helmholtz type equation:

$$
-\nabla \cdot[D(\vec{r}) \nabla \Phi(\vec{r}, \omega)]+\mu_{a}(\vec{r}) \Phi(\vec{r}, \omega)=q_{0}(\vec{r}, \omega),
$$

where $\Phi(\vec{r}, \omega)$ is the photon fluence rate at a position $\vec{r}, D$ $=1 /\left[3\left(\mu_{a}+\mu_{s}^{\prime}\right)\right]$ is the diffusion coefficient, $\mu_{a}$ is the absorption coefficient, $\mu_{s}^{\prime}$ is the scattering coefficient, $\omega$ is the angular modulation frequency, and $q_{0}(\vec{r}, \omega)$ is the source term. A Robin-type condition applies to the medium-applicator interface $\vec{r}_{0}$ as

$$
\Phi\left(\vec{r}_{0}, \omega\right)+2 D\left(\vec{r}_{0}\right) A \hat{n}_{0} \cdot \nabla \Phi\left(\vec{r}_{0}, \omega\right)=0,
$$

where $\hat{n}_{0}$ is the unit outward-pointing vector normal to the interface and $A$ is a coefficient determined by the reflective index mismatch across the boundary. By a phasor notation, $\Phi(\vec{r}, \omega)=I e^{j \theta}$, where $I$ and $\theta$ are the magnitude and phase of the photofluence rate, respectively, $\Phi(\vec{r}, \omega)$ can be implemented in logarithm as ${ }^{14}$

$$
\log [\Phi(\vec{r}, \omega)]=\log (I)+j \theta
$$

to yield a better scaled inverse problem for the reconstructions. ${ }^{15}$ An alternative form of Eq. (3), $\Psi(\vec{r}, \omega)$ $=\{\log (I), \theta\}$, will be used for the following analytics.

\section{II.A. Image reconstruction based on the measurements taken by individual source-detector-pairs}

We use $\left\langle S_{i}, D_{j}\right\rangle$ to denote a source-detector pair consisting of a source $i$ and a detector $j$ on the medium boundary, and $\left\{\left\langle S_{i}, D_{j}\right\rangle\right\}$ to indicate the ensemble of all pairs of sourcedetector. The conventional objective function of the DOT inverse problem is thereby ${ }^{13}$

$$
\|\chi\|^{2}=\left\|\left[\Psi_{\left\{\left\langle S_{i}, D_{j}\right\rangle\right\}}\right]_{m}-\left[\Psi_{\left\{\left\langle S_{i}, D_{j}\right\rangle\right\}}(\mu)\right]_{c}\right\|^{2},
$$

where $\mu$ specifies $\mu_{a}$ and $D$, the subscripts $m$ and $c$ represent, respectively, the "measurement" and the "calculation," and $\chi$ is the data-model misfit. The objective function in Eq. (4) is iteratively minimized by updating the calculated photon fluence rate using the following form, which ignores the higher 
order terms (order $\geq 2$ ) in Taylor series expansion around the $\mu$ values of the previous iteration:

$$
\left[\Psi\left(\mu_{n}\right)\right]_{c} \approx\left[\Psi\left(\mu_{n-1}\right)\right]_{c}+J \delta \mu_{n},
$$

where the subscripts $n$ and $n-1$ are the iteration numbers, and $\delta$ is the difference between the referred value at current and the previous iterations as $\delta \mu_{n}=\mu_{n}-\mu_{n-1}$. The $J$ in Eq. (5) is the Jacobian, or called the sensitivity matrix, which is the first order derivative of the measurement quantities with respect to the optical properties.

Rearranging Eq. (4) using Eq. (5) gives

$$
\begin{aligned}
\chi_{n} & =\Psi_{m}-\Psi_{c}\left(\mu_{n}\right) \approx \Psi_{m}-\Psi_{c}\left(\mu_{n-1}\right)-J \delta \mu_{n} \\
& =\chi_{n-1}-J \delta \mu_{n} .
\end{aligned}
$$

The reconstruction process, which is to iteratively compute the change of the objective function with respect to the change of the optical property until the change of the former reaches a predetermined stopping criterion, is equivalent to solving

$$
\frac{\partial(\chi)^{2}}{\partial \mu}=\frac{\partial\left(\Psi_{m}-\Psi_{c}\right)^{2}}{\partial \mu}=-2\left(\frac{\partial \Psi_{c}}{\partial \mu}\right) \chi=-2 \cdot J^{T} \chi=0 .
$$

Substituting Eq. (6) to Eq. (7) at the $n$th iteration gives

$$
J^{T} \cdot\left(\chi_{n-1}-J \cdot \delta \mu_{n}\right)=0 \text {. }
$$

Therefore, the update of the optical properties at the $n$th iteration is

$$
\delta \mu_{n}=\left(J^{T} J\right)^{-1} J^{T} \chi_{n-1} .
$$

To facilitate a better conditioning of the inversion of $J^{\mathrm{T}} J$ in Eq. (8b), a Levenberg-Marquardt scheme implements a diagonal regularization factor $\lambda$ in the form of

$$
\delta \mu_{n}=\left(J^{T} J+\lambda I\right)^{-1} J^{T} \chi_{n-1} .
$$

The $\lambda$ value is typically step-wisely adjusted, ${ }^{13}$ i.e., the $\lambda$ value at each step of iteration is reduced with respect to that in the immediate previous step (an empirical damping factor of 1.78 is used in this work).

We denote $\mathrm{N}$ as the total number of the spatial elements for which the updating of the optical properties is performed,
"NoS" as the number of source channels, and "NoD" as the number of detector channels. Then the complete Jacobian matrix, of FD measurements, amounts to the form of

$$
\begin{aligned}
& J_{[\mathrm{NoD} * \mathrm{NoS} * 2] \times[\mathrm{N} * 2]}=\left[\begin{array}{cc}
{\left[\frac{\partial \log (I)}{\partial \mu_{a}}\right]_{[\mathrm{NoD} * \mathrm{NoS}] \times \mathrm{N}}} & {\left[\frac{\partial \log (I)}{\partial D}\right]_{[\mathrm{NoD} * \mathrm{NoS}] \times \mathrm{N}}} \\
{\left[\frac{\partial \theta}{\partial \mu_{a}}\right]_{[\mathrm{NoD} * \mathrm{NoS}] \times \mathrm{N}}} & {\left[\frac{\partial \theta}{\partial D}\right]_{[\mathrm{NoD} * \mathrm{NoS}] \times \mathrm{N}}}
\end{array}\right],
\end{aligned}
$$

wherein each of the four blocks of the right-hand-side has (NoD*NoS) rows and $\mathrm{N}$ columns. In the following analytics, however, we will consider only the upper-left block of the Jacobian of Eq. (9a) for compactness. The upper-left block of the Jacobian may conform to the simplest case of recovering the absorption coefficients by using intensity information as could be rendered by $\mathrm{CW}$ measurements. However, the analytical formulations of the GSD method discussed for the CW case can be straightforwardly extended to recovering both absorption and scattering properties, which is not impractical by using only $\mathrm{CW}$ measurements, ${ }^{16-18}$ or to recovering both absorption and scattering properties by using FD measurements that include phase information. The extensions involve either doubling the numbers of columns or doubling the numbers of both columns and rows.

In the remaining analytics, as we will consider $J$ $=\left[\partial \log (I) / \partial \mu_{a}\right]_{[\mathrm{NoD} * \mathrm{NoS}] \times \mathrm{N}}, \log (I)$ is to be replaced by $\psi$, and $\mu_{a}$ by $\mu$, for clarity. The remaining analytics will also specify that $\mathrm{NoS}=8$ and $\mathrm{NoD}=8$, to conform to the geometry (Fig. 3) involved later in the simulation and experimental studies. With these customizations, the Jacobian matrix becomes

$$
J^{\diamond}=\left[\frac{\partial \psi}{\partial \mu^{\diamond}}\right]_{64 \times N}=\left[\begin{array}{llll}
J^{1} & J^{2} & \ldots \ldots & J^{N}
\end{array}\right],
$$

where $J^{\diamond}$ and $\mu^{\diamond}, \diamond=\{1: N\}$, are, respectively, the sub-matrix of $J$ and the $\mu$ associated with the " $\nabla$ th" spatial element. $J^{\diamond}$ has a matrix dimension of $64 \times 1$, and is shown explicitly as

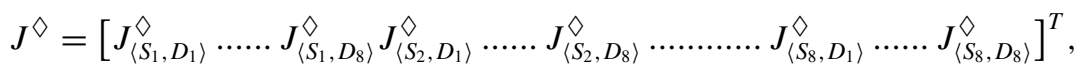

where

$$
J_{\left\langle S_{i}, D_{j}\right\rangle}^{\diamond}=\frac{\partial \Psi_{\left\langle S_{i}, D_{j}\right\rangle}}{\partial \mu \diamond} \quad i=\{1,2, \ldots . .8\} ; j=\{1,2, \ldots \ldots 8\} ; \diamond=\{1: N\}
$$

is the sensitivity of the measurement by the source-detectorpair $\left\langle S_{i}, D_{j}\right\rangle$ with respect to the " $\diamond$ th" spatial element.

The DOT measurement corresponding to an individual source-detector-pair is conceptually illustrated in Fig. 1. Figure 1(a) shows the measurement by the pair of source- detector $\left\langle S_{i}, D_{j}\right\rangle$ in response to a single object with a positive absorption contrast over the background at varied depths (either depth 1 or 2). The thinner solid curve within the medium indicates the hypothetical depth-dependence of the measurement sensitivity, i.e., $J_{\left\langle S_{i}, D_{j}\right\rangle}^{\diamond}$ of Eq. (10b) evaluated for all $\diamond$ 
elements located along the specific depth direction. The solid boxes at the right edge and pointed by the framed arrows represent the change of the measurements between without the object and with the object at the two depths. The signal change corresponding to the object at depth 1 would be greater than that corresponding to the identical object at depth 2 , owing to the variation of the measurement sensitivity with respect to depth. Figure 1(b) illustrates the effect of applying a series of regularization factors that decreases in strength along the depth (dashed curve), such as the one achieved by SVR approach. ${ }^{8}$ The outcome of this depth-adapted "regularization" is equivalent to suppressing the change of the datamodel misfit with respect to the same amount of update to the optical property at regions of higher measurement sensitivity. The regularization results in an overall reconstruction response (the thicker solid curve) that varies less with respect to the depth. On the other hand, Fig. 1(c) illustrates the effect of applying a sensitivity-weighting matrix that increases in strength along the depth (dashed curve), as is reported by the DCA approach. ${ }^{2}$ The outcome of this "weighting" approach is to elevate the change of the data-model misfit with respect to the same amount of update to the optical property in regions with less measurement sensitivity, which also results in an overall reconstruction response (the thicker solid curve) of varying less upon the depth.

\section{II.B. Image reconstruction based on paired measurements taken by source-sharing source-detector-pairs}

Figure 2 conceptually illustrates the measurements taken by two source-detector-pairs that share the source. The solid curve and the dashed curve represent hypothetical depthsensitivities of the measurements by $\left\langle S_{i}, D_{j}\right\rangle$ and $\left\langle S_{i}, D_{j+k}\right\rangle$, respectively, and $k$ is the difference between the serial numbers of the detectors in the two source-detector-pairs. These sensitivity values are $J_{\left\langle S_{i}, D_{j}\right\rangle}^{\diamond}$ and $J_{\left\langle S_{i}, D_{j+k}\right\rangle}^{\diamond}$, respectively, evaluated for the same set of $\diamond$ elements located along the specific depth direction. The solid boxes at the right edge and pointed by the framed arrows represent the change of the respective measurements by $\left\langle S_{i}, D_{j}\right\rangle$ and $\left\langle S_{i}, D_{j+k}\right\rangle$ without and with the object at the two depths. The signal change corresponding to either $\left\langle S_{i}, D_{j}\right\rangle$ and $\left\langle S_{i}, D_{j+k}\right\rangle$ for the object at depth 1 would be greater than that at depth 2 for the sensitivities varying with respect to depth as shown; however, the relative sensitivity between $\left\langle S_{i}, D_{j}\right\rangle$ and $\left\langle S_{i}, D_{j+k}\right\rangle$ for the same object at different depths varies much less than the individual sensitivity of $\left\langle S_{i}, D_{j}\right\rangle$ or $\left\langle S_{i}, D_{j+k}\right\rangle$ does. For the special case of the detector $D_{j}$ and $D_{j+k}$ being symmetric to the source $S_{i}$, and an object located at a position symmetric to the detector $D_{j}$ and $D_{j+k}$, the relative sensitivity between $\left\langle S_{i}, D_{j}\right\rangle$ and $\left\langle S_{i}, D_{j+k}\right\rangle$ will be the same with respect to the depth of the object, regardless of the depth-variation of the individual sensitivities of $\left\langle S_{i}, D_{j}\right\rangle$ or $\left\langle S_{i}, D_{j+k}\right\rangle$, for the object in an otherwise homogenous medium. These hypothetical analyses suggest that the objective function using the paired measurements taken by source-sharing source-detector-pairs could be insensitive to the depth of the object to be recovered, when comparing to

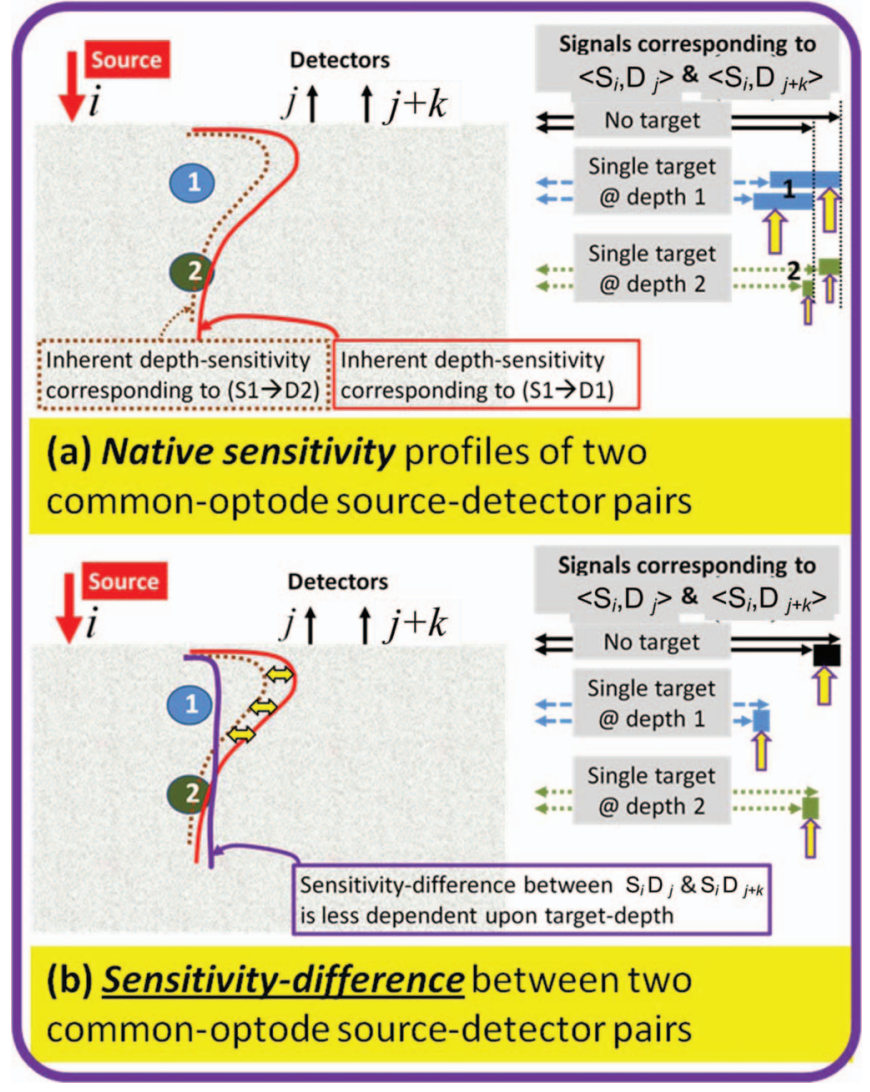

FIG. 2. Illustration of DOT image reconstruction approaches that utilizes the paired measurements from two source-sharing source-detector-pairs. The solid curve represents the native sensitivity profile of $\left\langle S_{i}, D_{j}\right\rangle$, the dashed curve represents the native sensitivity profile of $\left\langle S_{i}, D_{j+k}\right\rangle$. The framed dual-ended arrows indicate the difference between the sensitivity of the two source-detector-pairs. The framed arrows indicate the difference between the signal responses measured between the two source-detector-pairs. (a) The signal responses by either of the two same-source source-detector pairs $\left\langle S_{i}\right.$, $\left.D_{j}\right\rangle$ and $\left\langle S_{i}, D_{j+k}\right\rangle$ to an object located at different depths are different; (b) The relative differences between the signal responses of $\left\langle S_{i}, D_{j}\right\rangle$ and $\left\langle S_{i}, D_{j+k}\right\rangle$ to the same object at different depths are different, yet the difference between the sensitivity profiles of the two source-detector-pairs are less depthdependent.

the objective function using the unpaired measurements taken by individual source-detector-pairs.

We analyze the forward-pairing between two sourcesharing source-detector pairs, i.e., $\left\langle S_{i}, D_{j}\right\rangle$ and $\left\langle S_{i}, D_{m}\right\rangle$ with $j$ $<m$. The relative sensitivity of the measurements by $\left\langle S_{i}, D_{j}\right\rangle$ and $\left\langle S_{i}, D_{m}\right\rangle$ with respect to the $\diamond$ th element is

$$
\begin{aligned}
\frac{\partial\left[\Psi_{\left\langle S_{i}, D_{j}\right\rangle}-\Psi_{\left\langle S_{i}, D_{m}\right\rangle}\right]}{\partial \mu \diamond} & =\frac{\partial \Psi_{\left\langle S_{i}, D_{j}\right\rangle}}{\partial \mu^{\diamond}}-\frac{\partial \Psi_{\left\langle S_{i}, D_{m}\right\rangle}}{\partial \mu} \\
& =J_{\left\langle S_{i}, D_{j}\right\rangle}^{\diamond}-J_{\left\langle S_{i}, D_{m}\right\rangle}^{\diamond}=\tilde{J}_{\left\langle S_{i}, D_{j}, D_{m}\right\rangle}^{\diamond}
\end{aligned}
$$

The complete forward-pairing with respect to $\left\langle S_{1}, D_{1}\right\rangle$ composes $\tilde{J}_{\left\langle S_{i}, D_{j}, D_{m}\right\rangle}^{\diamond}$ with $i=1, j=1, m=\{2: 8\}$, which has the 
following explicit form:

$$
\left[\begin{array}{c}
\tilde{J}_{\left\langle S_{1}, D_{1}, D_{2}\right\rangle}^{\diamond} \\
\tilde{J}_{\left\langle S_{1}, D_{1}, D_{3}\right\rangle} \\
\tilde{J}_{\left\langle S_{1}, D_{1}, D_{4}\right\rangle}^{\diamond} \\
\tilde{J}_{\left\langle S_{1}, D_{1}, D_{5}\right\rangle}^{\diamond} \\
\tilde{J}_{\left\langle S_{1}, D_{1}, D_{6}\right\rangle} \\
\tilde{J}_{\left\langle S_{1}, D_{1}, D_{7}\right\rangle} \\
\tilde{J}_{\left\langle S_{1}, D_{1}, D_{8}\right\rangle}^{\diamond}
\end{array}\right]=\left[\begin{array}{cccccccc}
1 & -1 & 0 & 0 & 0 & 0 & 0 & 0 \\
1 & 0 & -1 & 0 & 0 & 0 & 0 & 0 \\
1 & 0 & 0 & -1 & 0 & 0 & 0 & 0 \\
1 & 0 & 0 & 0 & -1 & 0 & 0 & 0 \\
1 & 0 & 0 & 0 & 0 & -1 & 0 & 0 \\
1 & 0 & 0 & 0 & 0 & 0 & -1 & 0 \\
1 & 0 & 0 & 0 & 0 & 0 & 0 & -1
\end{array}\right]\left[\begin{array}{c}
J_{\left\langle S_{1}, D_{1}\right\rangle}^{\diamond} \\
J_{\left\langle S_{1}, D_{2}\right\rangle} \\
J_{\left\langle S_{1}, D_{3}\right\rangle}^{\diamond} \\
J_{\left\langle S_{1}, D_{4}\right\rangle}^{\diamond} \\
J_{\left\langle S_{1}, D_{5}\right\rangle}^{\diamond} \\
J_{\left\langle S_{1}, D_{6}\right\rangle}^{\diamond} \\
J_{\left\langle S_{1}, D_{7}\right\rangle}^{\diamond} \\
J_{\left\langle S_{1}, D_{8}\right\rangle}^{\diamond}
\end{array}\right] .
$$

Equation (11b) can be simplified to the form of

$$
\begin{aligned}
{\left[\tilde{J}_{\left\{\left\langle S_{1}, D_{1}, D_{2: 8}\right\}\right.}^{\diamond}\right]_{7 \times 1}=} & {\left[\operatorname{Diff}_{\left\langle S_{1}, D_{1}, D_{2: 8}\right\rangle}^{\diamond}\right]_{7 \times 8} } \\
& \times\left[\begin{array}{lllll}
J_{\left\langle S_{1}, D_{1}\right\rangle}^{\diamond} & J_{\left\langle S_{1}, D_{2}\right\rangle}^{\diamond} & \ldots \ldots & J_{\left\langle S_{1}, D_{8}\right\rangle}
\end{array}\right]^{T},
\end{aligned}
$$

where the [Diff] matrix performing the forward-pairing differentiation of the native sensitivity values is called the GSD operation matrix. The [Diff] matrix of Eq. (11c) that is associated with $\left\langle S_{1}, D_{1}\right\rangle$ has a dimension of $7 \times 8$, whereas similar complete forward-pairing GSD operations to $\left\langle S_{1}, D_{j}\right\rangle$, $j=\{2: 7\}$, will generate respective [Diff] matrix that has a dimension of $(8-j) \times 8$, as explicitly listed in Table I. Note that none of the empty blocks in Table I contribute to the differentiation (i.e., they are the "zero" elements in the [Diff] matrix). Each set of the blocks in Table I associated with the same starting source-detector-pair $\left\langle S_{1}, D_{\mathrm{j}}\right\rangle, j=\{1: 7\}$, in the forward-pairing GSD operation, can be expressed in a general form of

$$
\begin{aligned}
& {\left[\tilde{J}_{\left\{\left\langleS_{1}, D_{j}, D_{(j+1): 8}\right.\right.}^{\diamond}\right]_{(8-j) \times 1} } \\
= & {\left[\operatorname{Diff}_{\left\langle S_{1}, D_{j}, D_{(j+1): 8}\right\rangle}\right]_{(8-j) \times 8} } \\
& \times\left[\begin{array}{llll}
J_{\left\langle S_{1}, D_{1}\right\rangle}^{\diamond} & J_{\left\langle S_{1}, D_{2}\right\rangle}^{\diamond} & \ldots \ldots & J_{\left\langle S_{1}, D_{8}\right\rangle}^{\diamond}
\end{array}\right]_{j=\{1: 7\}} .
\end{aligned}
$$

The complete set of the blocks in Table I represents the forward-pairing GSD operation on all source-detector-pairs that share the source 1. The complete forward-pairing GSD operation on all source-detector-pairs that share each of the other seven sources will have a structure identical to the one shown in Table I, with only a change in the source channel. Therefore, the complete set of forward-pairing GSD operations to all source-detector-pairs that share the source $S_{i}$, $i=\{1: 8\}$, can be represented by $\tilde{J}_{\left\{\left\langle S_{i}, D_{j}, D_{m}\right\rangle\right\}}^{\diamond}$, or simplified as $\tilde{J}_{\left\{S_{i}\right\}}^{\diamond}$ of the following:

$$
\begin{aligned}
{\left[\tilde{J}_{\left\{S_{i}\right\}}^{\diamond}\right]_{28 \times 1}=} & {\left[\operatorname{Diff}_{\left\{S_{i}\right\}}^{\diamond}\right]_{28 \times 8} } \\
& \times\left.\left[\begin{array}{lllll}
J_{\left\langle S_{i}, D_{1}\right\rangle}^{\diamond} & J_{\left\langle S_{i}, D_{2}\right\rangle}^{\diamond} & \ldots . . & J_{\left\langle S_{i}, D_{8}\right\rangle}^{\diamond}
\end{array}\right]^{T}\right|_{i=\{1: 8\}} .
\end{aligned}
$$

The complete $\left[\tilde{J}_{\left\{S_{i}\right\}}^{\diamond}\right]_{28 \times 1}$ for $i=\{1: 8\}$ are presented in Table II, of which each block along the diagonal is identical to Table I in terms of the structure except a change to the source number $i$. Table II follows the matrix form of

$$
\left[\tilde{J}^{\diamond}\right]_{224 \times 1}=\left[\operatorname{Diff}^{\diamond}\right]_{224 \times 64}\left[J^{\diamond}\right]_{64 \times 1} \text {. }
$$

It is worthy to present the counterparts to Eqs. (12a)-(12c) for general NoS and NoD:

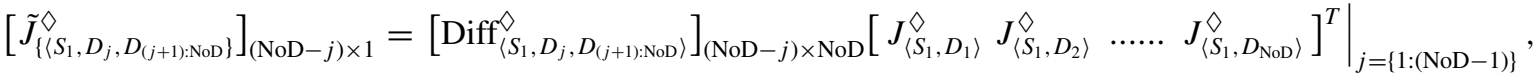

$$
\begin{aligned}
& {\left[\tilde{J}_{\left\{S_{i}\right\}}^{\diamond}\right]_{[(\mathrm{NoD}-1) * \mathrm{NoD} / 2] \times 1}=\left.\left[\operatorname{Diff}_{\left\{S_{i}\right\}}^{\diamond}\right]_{[(\mathrm{NoD}-1) * \mathrm{NoD} / 2] \times \mathrm{NoD}}\left[J_{\left\langle S_{i}, D_{1}\right\rangle}^{\diamond} J_{\left\langle S_{i}, D_{2}\right\rangle}^{\diamond \ldots . .} J_{\left\langle S_{i}, D_{\mathrm{NoD}}\right\rangle}^{\diamond}\right]^{T}\right|_{i=\{1: \mathrm{NoS}\}},} \\
& {\left[\tilde{J}^{\diamond}\right]_{[(\mathrm{NoD}-1) * \mathrm{NoD} / 2 * \mathrm{NoS}] \times 1}=\left[\mathrm{Diff}{ }^{\diamond}\right]_{[(\mathrm{NoD}-1) * \mathrm{NoD} / 2 * \mathrm{NoS}] \times[\mathrm{NoD} * \mathrm{NoS}]}\left[J^{\diamond}\right]_{[\mathrm{NoD} * \mathrm{NoS}] \times 1} .}
\end{aligned}
$$

By applying Eqs. (12c) or (13c) to all $\mathrm{N}$ spatial elements, a matrix transformation to the native sensitivity $J$ by a complete and nonredundant forward-pairing GSD-operator [Diff] follows as

$$
\tilde{J}=[\text { Diff }] J,
$$

where the dimension of $\tilde{J}$ is $[(\mathrm{NoD}-1) * \mathrm{NoD} / 2 * \mathrm{NoS}] \times 1$ $\times \mathrm{N}$, that of [Diff] is $[(\mathrm{NoD}-1) * \mathrm{NoD} / 2 * \mathrm{NoS}] \times$ $[\mathrm{NoS} * \mathrm{NoD}] \times \mathrm{N}$, and that of $J$ is $[\mathrm{NoS} * \mathrm{NoD}] \times 1 \times \mathrm{N}$. It is noticed that the matrix multiplication in Eq. (14) is performed for the first two dimensions of the three matrices.
The third dimension $\mathrm{N}$ indicates that the matrix multiplication described by Eq. (13) is repeated for each of the $\mathrm{N}$ elements. Therefore, the native Jacobian matrix is presented in the form of $[\mathrm{NoS} * \mathrm{NoD}] \times 1 \times \mathrm{N}$ rather than its original form of $[\mathrm{NoS} * \mathrm{NoD}] \times \mathrm{N}$.

By using the GSD-operation of Eq. (14), the objective function for reconstruction changes from the one of Eq. (4) to

$$
\begin{aligned}
\|\tilde{\chi}\|^{2} & =\left\|[\tilde{\Psi}]_{m}-[\tilde{\Psi}]_{c}\right\|^{2}=\|[\text { Diff }][\Psi]_{m}-[\text { Diff }][\Psi]_{c} \|^{2} \\
& =\left[\Psi_{m}-\Psi_{c}\right]^{T}[\text { Diff }]^{T}[\text { Diff }]\left[\Psi_{m}-\Psi_{c}\right] .
\end{aligned}
$$


TABLE I. Illustration of the iterative assembly of the geometric-difference of the sensitivities associated with source-detector channels sharing the source channel 1. Note: the blank cells are zeros. The bold italic characters indicate the sign flipping. The bold numbers will cancel the bold italic numbers during the column summation, leaving only the rows highlighted in green.

\begin{tabular}{|c|c|c|c|c|c|c|c|c|c|}
\hline Forward-paring with respect to & For the $\triangleleft$ th element & $J_{\left\langle S_{1}, D_{1}\right\rangle}$ & $J_{\left\langle S_{1}, D_{2}\right\rangle}$ & $J_{\left\langle S_{1}, D_{3}\right\rangle}$ & $J_{\left\langle S_{1}, D_{4}\right\rangle}$ & $J_{\left\langle S_{1}, D_{5}\right\rangle}$ & $J_{\left\langle S_{1}, D_{6}\right\rangle}$ & $J_{\left\langle S_{1}, D_{7}\right\rangle}$ & $J_{\left\langle S_{1}, D_{8}\right\rangle}$ \\
\hline \multirow[t]{7}{*}{$\left\langle S_{1}, D_{1}\right\rangle 7$ rows } & $\tilde{J}_{\left\langle S_{1}, D_{1}, D_{2}\right\rangle}$ & 1 & -1 & & & & & & \\
\hline & $\tilde{J}_{\left\langle S_{1}, D_{1}, D_{3}\right\rangle}$ & 1 & & -1 & & & & & \\
\hline & $\tilde{J}_{\left\langle S_{1}, D_{1}, D_{4}\right\rangle}$ & 1 & & & -1 & & & & \\
\hline & $\tilde{J}_{\left\langle S_{1}, D_{1}, D_{5}\right\rangle}$ & 1 & & & & -1 & & & \\
\hline & $\tilde{J}_{\left\langle S_{1}, D_{1}, D_{6}\right\rangle}$ & $1 \rightarrow(-1)$ & & & & & $-1 \rightarrow(1)$ & & \\
\hline & $\tilde{J}_{\left\langle S_{1}, D_{1}, D_{7}\right\rangle}$ & $1 \rightarrow(-1)$ & & & & & & $-1 \rightarrow(1)$ & \\
\hline & $\tilde{J}_{\left\langle S_{1}, D_{1}, D_{8}\right\rangle}$ & $1 \rightarrow(-1)$ & & & & & & & $-1 \rightarrow(1)$ \\
\hline \multirow[t]{6}{*}{$\left\langle S_{1}, D_{2}\right\rangle 6$ rows } & $\tilde{J}_{\left\langle S_{1}, D_{2}, D_{3}\right\rangle}$ & & 1 & -1 & & & & & \\
\hline & $\tilde{J}_{\left\langle S_{1}, D_{2}, D_{4}\right\rangle}$ & & 1 & & -1 & & & & \\
\hline & $\tilde{\tilde{J}}_{\left\langle S_{1}, D_{2}, D_{5}\right\rangle}$ & & 1 & & & -1 & & & \\
\hline & $\tilde{J}_{\left\langle S_{1}, D_{2}, D_{6}\right\rangle}$ & & 1 & & & & -1 & & \\
\hline & $\tilde{J}_{\left\langle S_{1}, D_{2}, D_{7}\right\rangle}$ & & $1 \rightarrow(-1)$ & & & & & $-1 \rightarrow(1)$ & \\
\hline & $\tilde{J}_{\left\langle S_{1}, D_{2}, D_{8}\right\rangle}$ & & $1 \rightarrow(-1)$ & & & & & & $-1 \rightarrow(1)$ \\
\hline \multirow[t]{5}{*}{$\left\langle S_{1}, D_{3}\right\rangle 5$ rows } & $\tilde{J}_{\left\langle S_{1}, D_{3}, D_{4}\right\rangle}$ & & & 1 & -1 & & & & \\
\hline & $\tilde{J}_{\left\langle S_{1}, D_{3}, D_{5}\right\rangle}$ & & & 1 & & -1 & & & \\
\hline & $\tilde{J}_{\left\langle S_{1}, D_{3}, D_{6}\right\rangle}$ & & & 1 & & & -1 & & \\
\hline & $\tilde{J}_{\left\langle S_{1}, D_{3}, D_{7}\right\rangle}$ & & & 1 & & & & -1 & \\
\hline & $\tilde{J}_{\left\langle S_{1}, D_{3}, D_{8}\right\rangle}$ & & & $1 \rightarrow(-1)$ & & & & & $-1 \rightarrow(1)$ \\
\hline \multirow[t]{4}{*}{$\left\langle S_{1}, D_{4}\right\rangle 4$ rows } & $\tilde{J}_{\left\langle S_{1}, D_{4}, D_{5}\right\rangle}$ & & & & 1 & -1 & & & \\
\hline & $\tilde{J}_{\left\langle S_{1}, D_{4}, D_{6}\right\rangle}$ & & & & 1 & & -1 & & \\
\hline & $\tilde{J}_{\left\langle S_{1}, D_{4}, D_{7}\right\rangle}$ & & & & 1 & & & -1 & \\
\hline & $\tilde{J}_{\left\langle S_{1}, D_{4}, D_{8}\right\rangle}$ & & & & 1 & & & & -1 \\
\hline \multirow[t]{3}{*}{$\left\langle S_{1}, D_{5}\right\rangle 3$ rows } & $\tilde{J}_{\left\langle S_{1}, D_{5}, D_{6}\right\rangle}$ & & & & & 1 & -1 & & \\
\hline & $\tilde{J}_{\left\langle S_{1}, D_{5}, D_{7}\right\rangle}$ & & & & & 1 & & -1 & \\
\hline & $\tilde{J}_{\left\langle S_{1}, D_{5}, D_{8}\right\rangle}$ & & & & & 1 & & & -1 \\
\hline \multirow[t]{2}{*}{$\left\langle S_{1}, D_{6}\right\rangle 2$ rows } & $\tilde{\boldsymbol{J}}_{\left\langle S_{1}, D_{6}, D_{7}\right\rangle}$ & & & & & & 1 & -1 & \\
\hline & $\tilde{J}_{\left\langle S_{1}, D_{6}, D_{8}\right\rangle}$ & & & & & & 1 & & -1 \\
\hline$\left\langle S_{1}, D_{7}\right\rangle 1$ row & $\tilde{J}_{\left\langle S_{1}, D_{7}, D_{8}\right\rangle}$ & & & & & & & 1 & -1 \\
\hline
\end{tabular}

Both $\tilde{\Psi}$ and $\tilde{\chi}$ are now column vectors that have $[\mathrm{NoS} * \operatorname{NoD} *(\mathrm{NoD}-1) / 2]$ rows. Accordingly, Eq. (8b) transforms to

$$
\begin{aligned}
\delta \mu_{n} & =\left(\tilde{J}^{T} \tilde{J}\right)^{-1} \times \tilde{J}^{T} \times \tilde{\chi}_{n-1} \\
& =\left(J^{T}[\mathrm{Diff}]^{T}[\mathrm{Diff}] J\right)^{-1} \times J^{T}[\mathrm{Diff}]^{T} \times[\text { Diff }] \chi_{n-1}
\end{aligned}
$$

and Eq. (8c) transforms to

$$
\delta \mu_{n}=\left(\tilde{J}^{T} \tilde{J}+\lambda I\right)^{-1} \times \tilde{J}^{T} \times \tilde{\chi}_{n-1} .
$$

\section{GEOMETRY AND METHODS OF RECONSTRUCTION INVOLVED}

\section{III.A. The geometry studied and the depth-dependence of the native sensitivity}

In this study we use a circular-array outward-imaging geometry as is shown in Fig. 3. This imaging geometry has an inner radius of $10 \mathrm{~mm}$ and an outer radius of $50 \mathrm{~mm}$, with eight sources and eight detectors evenly interspersed along the inner boundary. Note that the outer boundary of the reconstruction domain is not a physical bound-

\begin{tabular}{|c|c|c|c|c|c|c|c|c|}
\hline For the $\triangleleft$ th element & $J_{\left\{\left\langle S_{1}, D_{j}\right\rangle\right\}}$ & $J_{\left\{\left\langle S_{2}, D_{j}\right\rangle\right\}}$ & $J_{\left\{\left\langle S_{3}, D_{j}\right\rangle\right\}}$ & $J_{\left\{\left\langle S_{4}, D_{j}\right\rangle\right\}}$ & $J_{\left\{\left\langle S_{5}, D_{j}\right\rangle\right\}}$ & $J_{\left\{\left\langle S_{6}, D_{j}\right\rangle\right\}}$ & $J_{\left\{\left\langle S_{7}, D_{j}\right\rangle\right\}}$ & $J_{\left\{\left\langle S_{8}, D_{j}\right\rangle\right\}}$ \\
\hline$\left.\tilde{J}_{\left\{S_{1}\right\}}\right|_{28 \times 1}$ & Table I & & & & & & & \\
\hline$\left.\tilde{J}_{\left\{S_{2}\right\}}\right|_{28 \times 1}$ & & Table I & & & & & & \\
\hline$\left.\tilde{J}_{\left\{S_{3}\right\}}\right|_{28 \times 1}$ & & & Table I & & & & & \\
\hline$\left.\tilde{J}_{\left\{S_{4}\right\}}\right|_{28 \times 1}$ & & & & Table I & & & & \\
\hline$\left.\tilde{J}_{\left\{S_{5}\right\}}\right|_{28 \times 1}$ & & & & & Table I & & & \\
\hline$\left.\tilde{J}_{\left\{S_{6}\right\}}\right|_{28 \times 1}$ & & & & & & Table I & & \\
\hline$\left.\tilde{J}_{\left\{S_{7}\right\}}\right|_{28 \times 1}$ & & & & & & & Table I & \\
\hline$\left.\tilde{J}_{\left\{S_{8}\right\}}\right|_{28 \times 1}$ & & & & & & & & Table I \\
\hline
\end{tabular}
ary as is the inner boundary. However, the depth of the

TABLE II. Assembly of all source-detector pairs. Parameters in Table I are repeated for the $J_{\left\{\left\langle S_{i}, D_{j}\right\rangle\right\}}$ and $J_{\left\{\left\langle S_{i}, D_{j}, D_{j+k}\right\rangle\right\}}$. Note: blank cells are zeros. 


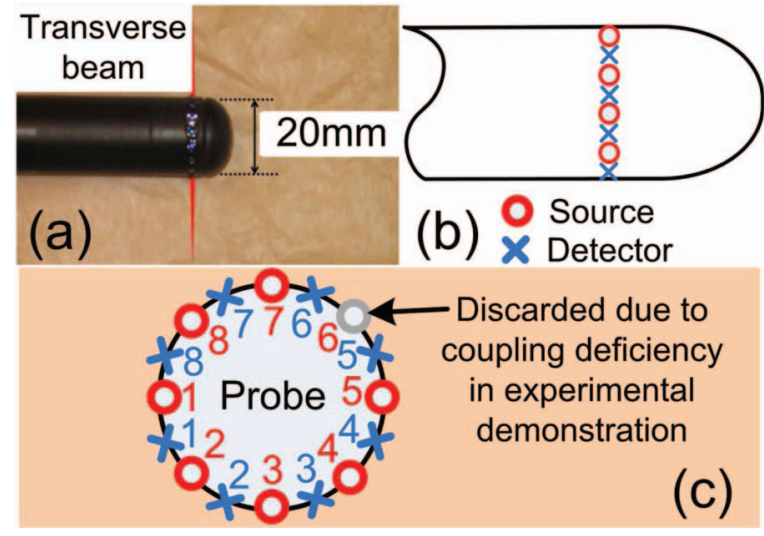

FIG. 3. Imaging geometry. (a) Photograph of the $20 \mathrm{~mm}$ diameter outwardimaging DOT applicator; (b) side-view; and (c) axial front view of the sourcedetector layout.

reconstruction domain warrants that treating the outer boundary as a physical one has minimal interference to the photon fluence rate in the useful imaging region proximal to the inner boundary. For this circular outward-imaging array what we concerned was reconstructing objects in the plane of the optode array as occurring to axial endorectal imaging; therefore, the introduced GSD method is evaluated primarily in a twodimensional geometry. Nonetheless, an example of applying GSD method to a simple three-dimensional geometry is also presented. The two-dimensional annular imaging domain applying to the geometry of Fig. 3 was discretized to a finite element mesh including 7708 evenly distributed nodes and 15040 elements. The display of the sensitivity distributions, the forward, and inverse computations were realized based on NIRFAST. ${ }^{14}$

The potential of GSD method in alleviating the sensitivity variation with respect to the imaging depth may be graph- ically appreciated by comparing the difference between the sensitivity profiles of two neighboring source-sharing sourcedetector-pairs as those shown in Fig. 4. Figures 4(a) and 4(b) display the native sensitivity distributions corresponding to $\left\langle S_{1}, D_{2}\right\rangle$ and $\left\langle S_{1}, D_{3}\right\rangle$, respectively. Plotted in Figs. 4(c)4(f) are the sensitivity values along the four radial directions within the overlapping area of the two sensitivity profiles. The two radial sensitivity profiles in all four directions within their common decaying-region are nearly parallel to each other in terms of the slopes of depth-degradation. The relatively even change of the two radial profiles over the decaying-region manifests that differentiating the two radial profiles will not produce a peak as prominent as presented by the individual radial profile. Note that at one place over the entire depth range the two radial profiles cross each other as shown in Figs. 4(d)4(f), indicating that the differentiation of the two radial profiles could encounter a sign change across a specific radial position. This sign change, however, is to be accompanied by the differentiation of the measurements by the two corresponding source-detector pairs when applying the GSD method, so the resulted product in Eq. (16) between the sensitivity and the measurement terms is likely to remain positive. Therefore, the crossing pattern that could occur in the GSD implementation does not necessarily cause the reversing of the contrast to be recovered, as will be demonstrated in Secs. IV-VI. Since the same principles apply to source-detector-pairs that share the detector, the remaining demonstrations are limited to sourcedetector-pairs that share the source.

\section{III.B. Choice of methods to which the GSD method is compared}

It is straightforward to evaluate the outcome of GSD-based DOT image reconstruction to that of a baseline method of DOT reconstruction that uses the native sensitivity profiles
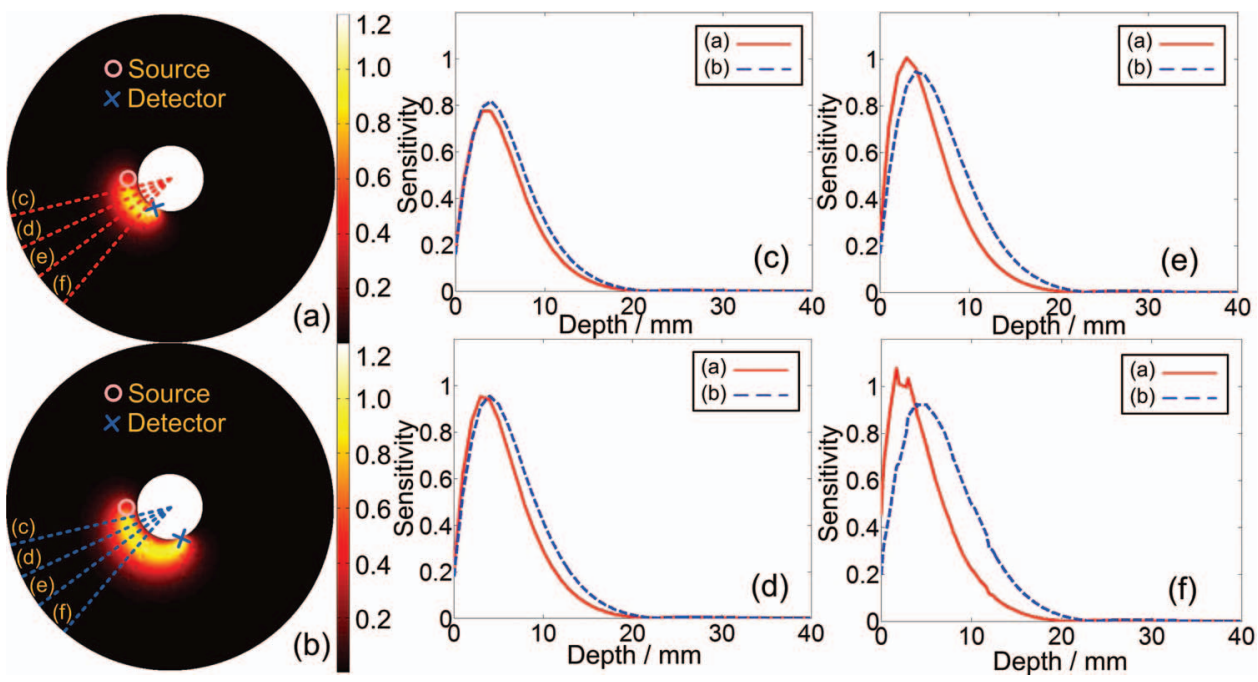

FIG. 4. The difference between the sensitivity profiles of two source-sharing source-detector-pairs in the imaging geometry corresponding to Fig. 3. (a) and (b) are the two-dimensional sensitivity profiles between two source-sharing source-detector-pairs. (c)-(f) include the sensitivity profiles along the marked directions for both source-detector-pairs. The two radial sensitivity profiles in all four directions within their common decaying-region are shown nearly parallel to each other in terms of the depth-degradation. 
but does not involve a depth-compensating scheme. On the other hand, it becomes imperative to compare the outcome of GSD method with that of methods including SVR or DCA that involves active compensation of the depth variation of the update function. Niu et al. ${ }^{19}$ have suggested that SVR method is not as robust as DCA method is in improving the image quality for deep objects in a planar-geometry. It is, therefore, anticipated that the SVR method would be less effective than the DCA method in dealing with the studied circular-array outward-imaging geometry of which the variation of the sensitivity with respect to imaging depth is much stronger than that of a planar geometry. The DCA method in Ref. 2 modifies the sensitivity matrix by a weighting scheme of $J_{R}=M^{\gamma} J$ to achieve an update function of the following:

$$
\delta \mu=\left[\left[J_{R}\right]^{T} J_{R}+\lambda I\right]^{-1}\left[J_{R}\right]^{T} \chi .
$$

The weighting implemented in $J_{R}$ is $M=\left\{\operatorname{diag}\left[\operatorname{MSV}\left(J_{l}\right)\right.\right.$, $\left.\left.\operatorname{MSV}\left(J_{l-1}\right), \ldots, \operatorname{MSV}\left(J_{2}\right), \operatorname{MSV}\left(J_{1}\right)\right]\right\}$, where $\operatorname{MSV}\left(J_{l}\right)$ stands for the maximum singular value of the sensitivity terms in $J_{l}$, the subscripts $\{1,2, \ldots, l\}$ denote the subgroups of $J$ in an artificially stratified layer $l$ over the depth, and $\gamma$ is a power factor. In Eq. (17) there are two parameters to optimize, the total number of layers $l$ and the power factor $\gamma$. We have tested this DCA method for the studied circular-array outward-imaging geometry in Appendix A, and concluded that the variation of the sensitivity with respect to the imaging depth of the studied geometry appears to be too strong for the DCA method to effectively compensate to the extent as having been demonstrated for the planar-geometry. ${ }^{2} \mathrm{We}$ hereby implement a reference-compensation method, which can be viewed as an enhanced DCA method, against which the GSD method will be evaluated. The update equation of this reference-compensation method is identical to Eq. (17) except that $J_{R}=M^{\mathrm{SVR}} J$, where $M^{\mathrm{SVR}}$ is a sensitivity compensation matrix that is exponentially regulated according to the radial depth of the spatial element, and is a square matrix that has all nonzero terms (totaling $\mathrm{N}$ ) located along its diagonal. For the studied circular-array outward-imaging geometry with an inner radius of $10 \mathrm{~mm}$ the diagonal elements of $M^{\mathrm{SVR}}$ are calculated for each of the $\mathrm{N}$ spatial elements with respect to their distance $(\rho)$ from the center of the geometry as

$$
\begin{aligned}
& M^{\mathrm{SVR}}(i, j) \\
& = \begin{cases}500 \exp ^{\left(\rho_{i}-10\right) /(R-10)} & \text { if }(i=j, \text { i.e., diagonal element }) \\
0 & \text { else }\end{cases}
\end{aligned}
$$

where $i$ and $j$ are the row and column indices of the $M^{\mathrm{SVR}}$ matrix, respectively.

\section{III.C. The effective sensitivity distributions and the optimization of the regularization parameters}

The effective sensitivity distribution over the entire imaging domain can be evaluated by summing the sensitivity terms corresponding to the same spatial element $\diamond$ and associated with all source-detector-pairs, i.e., $\sum\left[J_{\left\{\left\langle S_{i}, D_{j}\right\rangle\right\}}^{\diamond}\right]$ for the baseline method [Fig. 5(a)], $\sum\left[J_{R_{\left\{\left\langle S_{i}, D_{j}\right\}\right\}}}^{\diamond}\right]$ for the referencecompensation method [Fig. 5(b)], and $\sum\left[\tilde{J}_{\left\{\left\langle S_{i}, D_{j}, D_{m}\right\rangle\right]}^{\diamond}\right]$ for the GSD method [Fig. 5(c)]. The effective sensitivity distribution shown in Fig. 5(c) may appear rotationally asymmetric; however, the appearing asymmetry does not impair the actual rotational symmetry of the effective sensitivity. This can be illustrated by the following analysis that involves a slight rearrangement of the terms of GSD sensitivity matrix. Taking the sensitivity terms in Table I, for example, if the signs of all $\tilde{J}_{\left\{\left\langle S_{i}, D_{j}, D_{m}\right\rangle\right\}}$ terms that satisfy $m-j>4$ are reversed, the rows containing paired blocks with bold italic numbers such as " $\boldsymbol{l}$ " and " $-\boldsymbol{l}$ " will incur a sign change to the paired blocks, denoted by " $1 \rightarrow(-1)$ " and " $-1 \rightarrow(1)$ ", i.e., the reversing of the order of $J_{\left.\left\langle S_{i}, D_{j}\right\}\right\}}$ and $J_{\left.\left\langle S_{i}, D_{m}\right\rangle\right\}}$ in $\tilde{J}_{\left\{\left\langle S_{i}, D_{j}, D_{m}\right\}\right\}}$. Then if we perform a column-wise summation of all terms of Table I, those individual sensitivity terms of each column corresponding to the blocks containing bold numbers will cancel with the blocks containing bold Italic numbers. The uncancelled terms will contribute as the total effective sensitivity distribution imposed by $S_{1}$ :

$$
\begin{aligned}
\left.\sum\left[\tilde{J}_{\left\{\left\langle S_{i}, D_{j}, D_{m}\right\rangle\right\}}^{\diamond}\right]\right|_{i=1}= & \tilde{J}_{\left\langle S_{1}, D_{1}, D_{5}\right\rangle}+\tilde{J}_{\left\langle S_{1}, D_{2}, D_{6}\right\rangle} \\
& +\tilde{J}_{\left\langle S_{1}, D_{3}, D_{7}\right\rangle}+\tilde{J}_{\left\langle S_{1}, D_{4}, D_{8}\right\rangle} .
\end{aligned}
$$

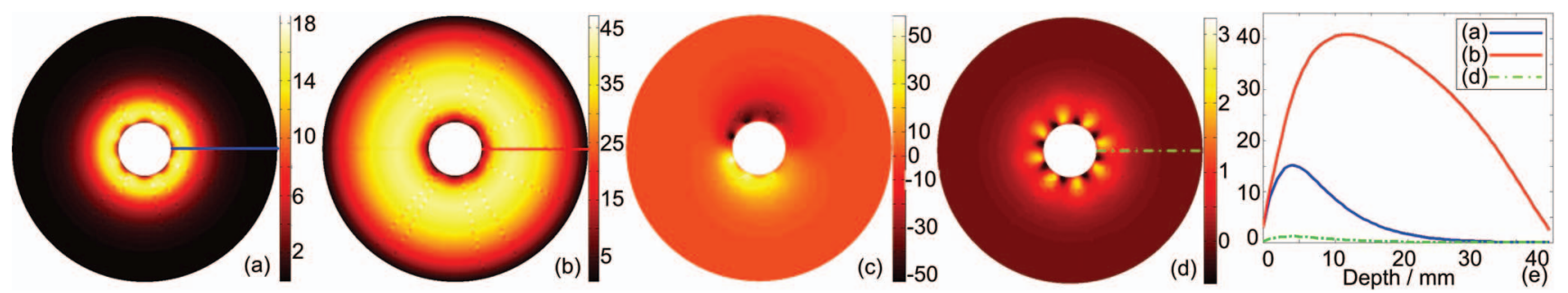

FIG. 5. Effective sensitivity $\left[\partial \log (I) / \partial \mu_{a}\right]$ distributions evaluated for the entire imaging domain. (Unit: $\mathrm{dB} / \mathrm{mm}^{-1}$ ) (a) The one corresponding to $J$ of the baseline method and Eq. (6). (b) The one corresponding to $J_{R}$ of the reference compensation method and Eq. (17). (c) The one corresponding to $\tilde{J}$ of the GSD method and Eq. (12). (d) The one corresponding to rearranged $\tilde{J}$ of the GSD method and Table III. The radial profiles of the effective sensitivity distributions of (a), (b), and (d) are plotted together in (e).The effective sensitivity of the baseline method varies greatly with respect to the depth and peaks at an approximate depth of $4 \mathrm{~mm}$. The reference-compensation method elevates the effective sensitivity in the entire depth and shifts the apparent peak of it to an approximate depth of $10 \mathrm{~mm}$, a much greater depth than that of baseline method. The effective sensitivity by the GSD method varies negligibly with respect to the depth and is negligibly small in strength comparing to the other two methods. 
TABLE III. Rearranged GSD matrix resulting in the effective sensitive profile of the one shown in Fig. 5(d). Bold italic numbers indicate the sign flippings.

\begin{tabular}{|c|c|c|c|c|c|c|c|c|c|}
\hline Forward-paring with respect to & For the $\diamond$-th element & $J_{\left\langle S_{1}, D_{1}\right\rangle}$ & $J_{\left\langle S_{1}, D_{2}\right\rangle}$ & $J_{\left\langle S_{1}, D_{3}\right\rangle}$ & $J_{\left\langle S_{1}, D_{4}\right\rangle}$ & $J_{\left\langle S_{1}, D_{5}\right\rangle}$ & $J_{\left\langle S_{1}, D_{6}\right\rangle}$ & $J_{\left\langle S_{1}, D_{7}\right\rangle}$ & $J_{\left\langle S_{1}, D_{8}\right\rangle}$ \\
\hline \multirow{4}{*}{$\left\langle S_{1}\right\rangle 4$ rows } & $\tilde{J}_{\left\langle S_{1}, D_{1}, D_{5}\right\rangle}$ & 1 & & & & -1 & & & \\
\hline & $\tilde{J}_{\left\langle S_{1}, D_{2}, D_{6}\right\rangle}$ & & 1 & & & & -1 & & \\
\hline & $\tilde{J}_{\left\langle S_{1}, D_{3}, D_{7}\right\rangle}$ & & & 1 & & & & -1 & \\
\hline & $\tilde{J}_{\left\langle S_{1}, D_{4}, D_{8}\right\rangle}$ & & & & 1 & & & & -1 \\
\hline \multirow[t]{4}{*}{$\left\langle S_{2}\right\rangle 4$ rows } & $-\tilde{J}_{\left\langle S_{2}, D_{1}, D_{5}\right\rangle}$ & $1 \rightarrow(-1)$ & & & & $-1 \rightarrow(1)$ & & & \\
\hline & $\tilde{J}_{\left\langle S_{2}, D_{2}, D_{6}\right\rangle}$ & & 1 & & & & -1 & & \\
\hline & $\tilde{J}_{\left\langle S_{2}, D_{3}, D_{7}\right\rangle}$ & & & 1 & & & & -1 & \\
\hline & $\tilde{J}_{\left\langle S_{2}, D_{4}, D_{8}\right\rangle}$ & & & & 1 & & & & -1 \\
\hline \multirow[t]{4}{*}{$\left\langle S_{3}\right\rangle 4$ rows } & $-\tilde{J}_{\left\langle S_{3}, D_{1}, D_{5}\right\rangle}$ & $1 \rightarrow(-1)$ & & & & $-1 \rightarrow(1)$ & & & \\
\hline & $-\tilde{J}_{\left\langle S_{3}, D_{2}, D_{6}\right\rangle}$ & & $1 \rightarrow(-1)$ & & & & $-1 \rightarrow(1)$ & & \\
\hline & $\tilde{J}_{\left\langle S_{3}, D_{3}, D_{7}\right\rangle}$ & & & 1 & & & & -1 & \\
\hline & $\tilde{J}_{\left\langle S_{3}, D_{4}, D_{8}\right\rangle}$ & & & & 1 & & & & -1 \\
\hline \multirow[t]{4}{*}{$\left\langle S_{4}\right\rangle 4$ rows } & $-\tilde{J}_{\left\langle S_{4}, D_{1}, D_{5}\right\rangle}$ & $1 \rightarrow(-1)$ & & & & $-1 \rightarrow(1)$ & & & \\
\hline & $-\tilde{J}_{\left\langle S_{4}, D_{2}, D_{6}\right\rangle}$ & & $1 \rightarrow(-1)$ & & & & $-1 \rightarrow(1)$ & & \\
\hline & $-\tilde{J}_{\left\langle S_{4}, D_{3}, D_{7}\right\rangle}$ & & & $1 \rightarrow(-1)$ & & & & $-1 \rightarrow(1)$ & \\
\hline & $\tilde{J}_{\left\langle S_{4}, D_{4}, D_{8}\right\rangle}$ & & & & 1 & & & & -1 \\
\hline \multirow[t]{4}{*}{$\left\langle S_{5}\right\rangle 4$ rows } & $-\tilde{\boldsymbol{J}}_{\left\langle S_{5}, D_{1}, D_{5}\right\rangle}$ & $1 \rightarrow(-1)$ & & & & $-1 \rightarrow(1)$ & & & \\
\hline & $-\tilde{J}_{\left\langle S_{5}, D_{2}, D_{6}\right\rangle}$ & & $1 \rightarrow(-1)$ & & & & $-1 \rightarrow(1)$ & & \\
\hline & $-\tilde{J}_{\left\langle S_{5}, D_{3}, D_{7}\right\rangle}$ & & & $1 \rightarrow(-1)$ & & & & $-1 \rightarrow(1)$ & \\
\hline & $-\tilde{J}_{\left\langle S_{5}, D_{4}, D_{8}\right\rangle}$ & & & & $1 \rightarrow(-1)$ & & & & $-1 \rightarrow(1)$ \\
\hline \multirow[t]{4}{*}{$\left\langle S_{6}\right\rangle 4$ rows } & $\tilde{J}_{\left\langle S_{6}, D_{1}, D_{5}\right\rangle}$ & 1 & & & & -1 & & & \\
\hline & $-\tilde{\boldsymbol{J}}_{\left\langle S_{6}, D_{2}, D_{6}\right\rangle}$ & & $1 \rightarrow(-1)$ & & & & $-1 \rightarrow(1)$ & & \\
\hline & $-\tilde{J}_{\left\langle S_{6}, D_{3}, D_{7}\right\rangle}$ & & & $1 \rightarrow(-1)$ & & & & $-1 \rightarrow(1)$ & \\
\hline & $-\tilde{J}_{\left\langle S_{6}, D_{4}, D_{8}\right\rangle}$ & & & & $1 \rightarrow(-1)$ & & & & $-1 \rightarrow(1)$ \\
\hline \multirow[t]{4}{*}{$\left\langle S_{7}\right\rangle 4$ rows } & $\tilde{J}_{\left\langle S_{7}, D_{1}, D_{5}\right\rangle}$ & 1 & & & & -1 & & & \\
\hline & $\tilde{J}_{\left\langle S_{7}, D_{2}, D_{6}\right\rangle}$ & & 1 & & & & -1 & & \\
\hline & $-\tilde{J}_{\left\langle S_{7}, D_{3}, D_{7}\right\rangle}$ & & & $1 \rightarrow(-1)$ & & & & $-1 \rightarrow(1)$ & \\
\hline & $-\tilde{J}_{\left\langle S_{7}, D_{4}, D_{8}\right\rangle}$ & & & & $1 \rightarrow(-1)$ & & & & $-1 \rightarrow(1)$ \\
\hline \multirow[t]{4}{*}{$\left\langle S_{8}\right\rangle 4$ rows } & $\tilde{J}_{\left\langle S_{8}, D_{1}, D_{5}\right\rangle}$ & 1 & & & & -1 & & & \\
\hline & $\tilde{J}_{\left\langle S_{8}, D_{2}, D_{6}\right\rangle}$ & & 1 & & & & -1 & & \\
\hline & $\tilde{J}_{\left\langle S_{8}, D_{3}, D_{7}\right\rangle}$ & & & 1 & & & & -1 & \\
\hline & $-\tilde{J}_{\left\langle S_{8}, D_{4}, D_{8}\right\rangle}$ & & & & $1 \rightarrow(-1)$ & & & & $-1 \rightarrow(1)$ \\
\hline
\end{tabular}

Repeating the forward-paring GSD operation for all sources, and reversing the signs of every associated $\tilde{J}_{\left\{\left\langle S_{i}, D_{j}, D_{m}\right\rangle\right\}}$ terms that satisfy $m-j>4$ as exemplified in Table I, the resulted complete effective sensitivity distribution becomes

$$
\begin{aligned}
\sum & {\left.\left[\tilde{J}_{\left\{\left\langle S_{i}, D_{i}, D_{m}\right\rangle\right\}}\right]\right|_{i=1: 8} } \\
= & \tilde{J}_{\left\{\left\langle S_{i}, D_{1}, D_{5}\right\rangle\right\}}+\tilde{J}_{\left\{\left\langle S_{i}, D_{2}, D_{6}\right\rangle\right\}}+\tilde{J}_{\left\{\left\langle S_{i}, D_{3}, D_{7}\right\rangle\right\}}+\left.\tilde{J}_{\left\{\left\langle S_{i}, D_{4}, D_{8}\right\rangle\right\}}\right|_{i=1: 8} \\
= & {\left[J_{\left\{\left\langle S_{i}, D_{1}\right\rangle\right\}}-J_{\left\{\left\langle S_{i}, D_{5}\right\rangle\right\}}\right]+\left[J_{\left\{\left\langle S_{i}, D_{2}\right\rangle\right\}}-J_{\left\{\left\langle S_{i}, D_{6}\right\rangle\right\}}\right] } \\
& +\left[J_{\left\{\left\langle S_{i}, D_{3}\right\rangle\right\}}-J_{\left\{\left\langle S_{i}, D_{7}\right\rangle\right\}}\right]+\left.\left[J_{\left\{\left\langle S_{i}, D_{4}\right\rangle\right\}}-J_{\left\{\left\langle S_{i}, D_{8}\right\rangle\right\}}\right]\right|_{i=1: 8} .
\end{aligned}
$$

Note that the sensitivity terms related to the detectors 1 to 4 are positive in the summation in Eq. (19b), whereas those related to detectors 5 to 8 are negative. Equation (19b) can effectively explain the asymmetry appeared in Fig. 5(c): (1) stronger sensitivity from 8 o'clock position counterclockwise to 4 o'clock position corresponds to the positive terms related to detectors 1 to 4 ; and (2) the weaker sensitivity from 2 o'clock position counterclockwise to 10 o'clock position corresponds to the negative terms related to detectors 5 to 8 . It should be noted that, all the sign- reversing or pair-flipping operations needed for reaching Eq. (19b) are linear. Therefore, the sign-reversing or pair-flipping of the sensitivity terms, and the corresponding measurement terms, does not alter the native rotational symmetry of the reconstruction. We thereby could further rearrange the sensitivity terms in Eq. (19b), by following the bolded sign-reversals detailed in Table III, to generate what is appearing to be a rotationally symmetric effective sensitivity distribution as that shown in Fig. 5(d). It should be clarified that these aforementioned rearranging operations to $\tilde{J}$ are presented only to demonstrate that the GSD operation does not alter the original symmetry of the measurement sensitivity, which for this studied geometry is a rotational-symmetry. The actual $\tilde{J}$ implemented to the simulations and experiments of Secs. IV-VI take the form of that specified by Eq. (13). Figure 5(e) then compares the effective distribution profiles of the native sensitivity $J$, the reference-compensating applied sensitivity $J_{R}$, and the rearranged GSD-operated sensitivity $\tilde{J}$, along the marked depth direction. It can be appreciated that the effective sensitivity profile of GSD method is nearly depthinvariant and insignificant comparing to the other two methods, whereas the effective sensitivity profile of the referencecompensation method peaks at a significantly increased depth 


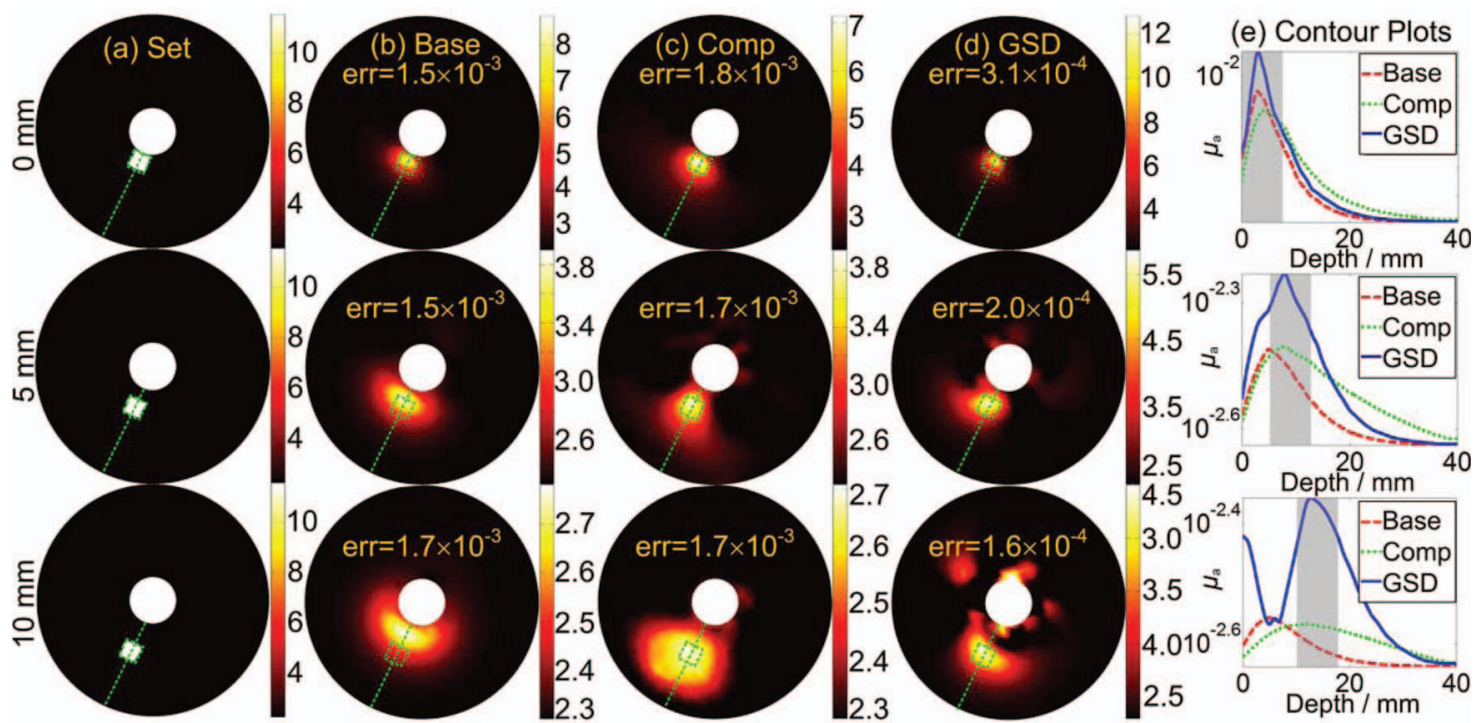

FIG. 6. Simulation results for single-object cases [unit used for columns (a)-(d): $10^{-3} \mathrm{~mm}^{-1}$, unit used for column (e): $\mathrm{mm}^{-1}$ ]. Column (a) set values; column (b) reconstruction based on the baseline method; column (c) reconstruction based on the reference-compensation method; column (d) reconstruction based on the GSD method; column (e) contour plots along the dotted lines marked in columns (b)-(d). The shaded areas in column (d) indicate the object locations corresponding to those marked in column (a). The "err"s in subfigures of columns (b)-(d) represent the standard deviation of the reconstruction errors averaged by the number of components in the objective function. Columns (c) and (d) show better depth-localization than (b). Column (d) has the best overall estimation of domain optical properties, albeit there are artifacts appearing in the case of $10 \mathrm{~mm}$ object-depth.

and is significantly stronger when comparing to that of the baseline method.

The strategy to step-wisely adjust the regularization factor $\lambda$ of Eq. (17) for the reference-compensation method is detailed in Appendix B. The tests have shown that an initial value of $\lambda=100$ and a step-wise damping rate of 1.78 are optimal for the reference-compensation method. The regularization factors $\lambda$ of Eq. (8c) for the baseline method should have been optimized individually; however, as the baseline method performs very poorly in the studied geometry as to impair the evaluation of the outcomes at different choices of the factor $\lambda$, the step-wise adjusting of the $\lambda$ for the reference-compensation method is applied directly to the baseline method. In all reconstructions the iteration stops when the change of the projection error between consecutive steps is less than $2 \%$.

\section{SIMULATION AND EXPERIMENTAL METHODS}

\section{IV.A. Simulation setup}

The optical heterogeneities employed for the simulation study were either a single cube that has a $7.5 \mathrm{~mm}$ side-length and $\mu_{a}=0.0115 \mathrm{~mm}^{-1}$ (five times of contrast over the background) or two such identical cubes separated azimuthally by $90^{\circ}$. The cubic-shaped objects were preferred for the fabrication handiness in the following experimental studies, on the other hand, a cube or sphere of $7.5 \mathrm{~mm}$ size may be insignificantly different to the experimental DOT system as its spatial resolution was low, of the order of 5 millimeters. The arrayfacing-edges of the cubic objects were placed 0,5 , and 10 $\mathrm{mm}$ away from the optical array, as shown in the column (a) of Figs. 6 and $8-10$. Gaussian noise of $1 \%$ was added to this set and all other sets of forward computation unless otherwise specified. The reconstructions based on the baseline method, the reference-compensation method, and the GSD method are presented side-by-side in the respective sections.

\section{IV.B. Experimental system and the actual number of optodes}

A DOT system rendering CW measurements ${ }^{21}$ was used to experimentally test the GSD method. The system to obtain the $\mathrm{CW}$ measurements contained a $4 \mathrm{~mW}$ wavelength-swept light source (Superlum BS-840-02) with a sweeping range of $838-853 \mathrm{~nm}$. The light was coupled to a $500 \mathrm{~mm}$ focal-length spectrometer (SpectroPro 500i, Princeton Instrument) with a grating of 1200 grooves $/ \mathrm{mm}$. The spectral-sweeping of the light was transformed to a spatial scanning of the light beam at the exit port of the spectrometer for sequential coupling to the 8 source-fiber channels of the circular-array outwardimaging DOT applicator of $20 \mathrm{~mm}$ in diameter. The light reemitting from the medium and reaching the 8 detector-fiber channels was acquired by a CCD mounted on another spectrometer (PIXIS 512 and SpectroPro 2300i, Princeton Instrument). The wavelength-sweeping was synchronized with the CCD exposure, at a data acquisition rate of 0.5 frame/s for the axial-imaging optical applicator to be placed in $1 \%$ intralipid solution $\left(\mu_{a}=0.0023 \mathrm{~mm}^{-1}\right.$ and $\left.\mu_{s}^{\prime}=1 \mathrm{~mm}^{-1}\right)$. The details of the method for calibrating the raw data can be found in Ref. 21.

The $20 \mathrm{~mm}$ applicator photographed in Fig. 3(a) was designed to house 8 source and 8 detector channels; however, one of the source channels suffered from missalignment of the micro-optical components and consequently very low coupling efficiency [marked in Fig. 3(c)] comparing to the 
rest 7 source channels. The measurements corresponding to this low-efficiency source channel had to be discarded. Accordingly, the simulated or experimental measurements were conducted on 7 source channels and 8 detector channels. The cubic-shaped object inclusions used for the experimental measurements were identical in sizes to those for simulations. However, the inclusions were made from black plastic materials of which the absorption contrast over the background was considerably higher than that in the simulation. The use of stronger-contrast object was found necessary to generate measureable signal change from the low power level $(4 \mathrm{~mW})$ of the light source, especially when the targets were deep.

\section{IV.C. A simulation of applying the GSD method to a simple three-dimensional geometry}

A simple three-dimensional planar reflectance geometry shown in Fig. 11 is used to exemplify the application of GSD method beyond the two-dimensional geometry of the aforementioned simulation and experimental studies. This example is conducted by simulation only, and involved the GSD method with respect to only the baseline method. The imaging geometry contains 18 sources and 18 detectors orderly arranged to the side of the medium, and the cubic imaging domain was discretized to 6466 nodes and 33591 elements. The optical properties of the background were $\mu_{a}=0.0023$ $\mathrm{mm}^{-1}$ and $\mu_{s}^{\prime}=1 \mathrm{~mm}^{-1}$, and a spherical inclusion of $5 \mathrm{~mm}$ in radius with $\mu_{a}=0.006 \mathrm{~mm}^{-1}$ and $\mu_{s}^{\prime}=1.1 \mathrm{~mm}^{-1}$ was located at a center depth of $10-30 \mathrm{~mm}$, at a step size of $5 \mathrm{~mm}$. The reconstruction algorithms and other parameters were identical to those used in the simulations in Sec. IV.A and IV.B.

\section{RESULTS}

\section{V.A. Simulation for single-object cases}

The simulation results of resolving single object by using the three reconstruction methods are shown in Figs. 6(b)-6(e)

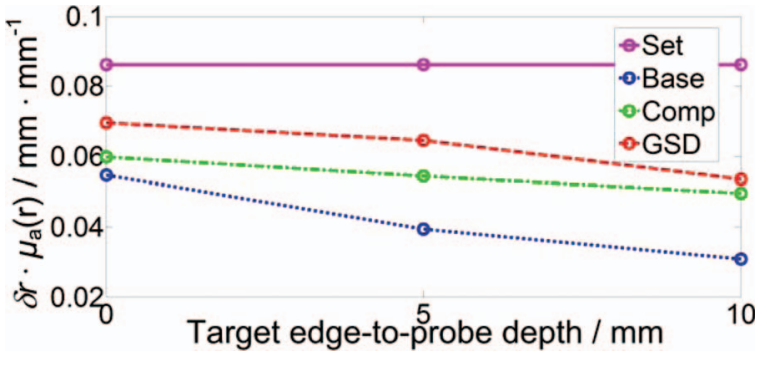

FIG. 7. Recovered object volume vs object depth. The curves represent the area covered by FWHM of the curves in column (e) in Fig. 6. Although all three methods show decreased area-within-the-FWHM profile as the depth increases, the change is the least by the GSD method, and the most by the baseline method.

along with the standard deviations of the reconstruction errors averaged by the number of components in the objective functions. The values and radial positions of the maximum recovered absorption coefficients in the object regions, as well as the mismatch between the set and reconstructed absorption distribution, are listed, respectively, for each simulation set in Table IV. It is observable in Figs. 6(b)-6(d) that the contours of the object are overestimated more in the azimuthal direction than in the radial direction. We thereby also included in Fig. 7 the areas covered by the full width at half maximum (FWHM) of the peak optical property of the recovered objects by the three methods.

By the baseline method the objects at three different depths are recovered at the same depth close to the optical array. By both the reference-compensation and GSD methods the objects are recovered at approximately the true depths, and it is appreciable that the azimuthal FWHM and the volume of the object resolved by the GSD method are closer to the set values than those by the reference-compensation method. The object optical properties were underestimated at greater depths by all three methods; however, the underestimation was the least by the GSD method. It is observed that artifacts do appear in GSD group at the $10 \mathrm{~mm}$ case. This is

TABLE IV. Simulation and experimental results for the reconstruction of single object. "Comp" stands for "reference-compensation.

\begin{tabular}{|c|c|c|c|c|c|}
\hline & e-depth and other relevant parameters & Set & Base & Comp & GSD \\
\hline \multirow[t]{4}{*}{$0 \mathrm{~mm}$} & Maximum $\mu_{a}\left(\mathrm{~mm}^{-1}\right)$ & 0.0115 & 0.0088 & 0.0071 & 0.013 \\
\hline & Radial position of maximum $\mu_{a}(\mathrm{~mm})$ & & 3.0 & 5.0 & 3.0 \\
\hline & Azimuthal FWHM (mm) & 7.5 & 9.7 & 11.3 & 7.0 \\
\hline & Averaged absorption map mismatch $\left(\mathrm{mm}^{-1}\right)$ & 0 & $1.89 \times 10^{-5}$ & $1.87 \times 10^{-5}$ & $1.80 \times 10^{-5}$ \\
\hline \multirow[t]{4}{*}{$5 \mathrm{~mm}$} & Maximum $\mu_{a}\left(\mathrm{~mm}^{-1}\right)$ & 0.0115 & 0.0039 & 0.0039 & 0.0059 \\
\hline & Radial position of maximum $\mu_{a}(\mathrm{~mm})$ & & 5.0 & 8.0 & 8.0 \\
\hline & Azimuthal FWHM (mm) & 7.5 & 20.0 & 19.6 & 14.4 \\
\hline & Averaged absorption map mismatch $\left(\mathrm{mm}^{-1}\right)$ & 0 & $1.81 \times 10^{-5}$ & $1.74 \times 10^{-5}$ & $1.72 \times 10^{-5}$ \\
\hline \multirow[t]{4}{*}{$10 \mathrm{~mm}$} & Maximum $\mu_{a}\left(\mathrm{~mm}^{-1}\right)$ & 0.0115 & 0.0028 & 0.0027 & 0.0044 \\
\hline & Radial position of maximum $\mu_{a}(\mathrm{~mm})$ & & 5.0 & 11.0 & 12.0 \\
\hline & Azimuthal FWHM (mm) & 7.5 & 26.6 & 32.9 & 19.7 \\
\hline & Averaged absorption map mismatch $\left(\mathrm{mm}^{-1}\right)$ & 0 & $1.87 \times 10^{-5}$ & $1.81 \times 10^{-5}$ & $1.69 \times 10^{-5}$ \\
\hline
\end{tabular}




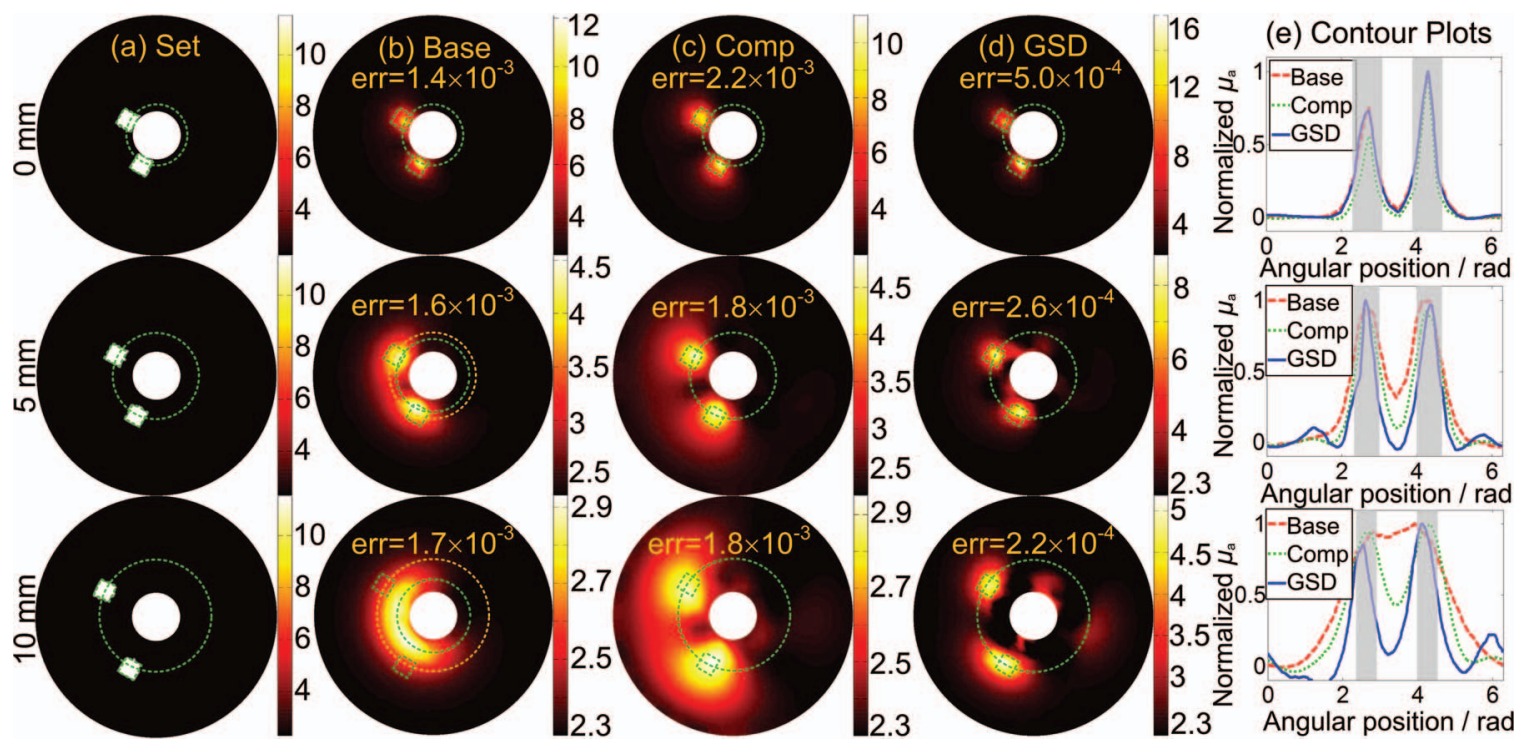

FIG. 8. Simulation results for dual-object cases [unit used for columns (a)-(d): $10^{-3} \mathrm{~mm}^{-1}$ ]. Column (a) set values; column (b) reconstruction based on the baseline method; column (c) reconstruction based on the reference-compensation method; column (d) reconstruction based on the GSD method; column (e) normalized contour plots along the dotted loops marked in columns (b)-(d). The shaded areas in column (e) indicate the object locations corresponding to those marked in column (a). Note that although the widths of the object remain constant for all three depth, the radial expansion represented by the shaded areas in column (d) decreases with respect to imaging depth. The dotted circles in column (d) indicate the object locations corresponding to those marked in column (a). The "err"s in subfigures of columns (b)-(d) represent the standard deviation of the reconstruction errors averaged by the number of components in the objective function. Column (d) has the best overall resolution of the two objects and the most accurate estimation of domain optical properties.

not unexpected, because as the native measurement sensitivity diminishes significantly with respect to the depth, the effectiveness of GSD at that depth is also going to be mitigated. Nonetheless, the object recovery by the GSD method is of better quality than by the other two methods, as analyzed in Table IV and Fig. 7. The area-within-the-FWHM decreases as the depth increases, suggesting degradation of the reconstruction quality, by all three methods, yet the degradation in the GSD method is the least. In the rest of studies results similar to Table IV and Fig. 7 were observed and not separately presented.

\section{V.B. Simulation for two-target cases}

The objects as aforementioned were two identical cubes with a $7.5 \mathrm{~mm}$ side-length and were azimuthally separated by $90^{\circ}$, as illustrated in Fig. 8(a). The imaging results are given in Figs. 8(b)-8(e). The $\mu_{a}$ values along the azimuthal contours across the recovered objects are displayed in column (e) of Fig. 8. By the baseline method the objects at three different depths were recovered at almost the same depth close to the optical array, and the two objects at the $10 \mathrm{~mm}$ depth were not distinguished. By both the reference-compensation and GSD methods the objects were recovered at the set positions, but the contour plots of Fig. 8(e) show that the GSD method outperforms the other one in terms of the object size, the distinguishing of the two objects, and the estimations of the object optical properties. These profiles also suggest the outperformance of the GSD method over the other two methods.

\section{V.C. Experimental results for single-object cases}

The experimental results for single-object cases are given in Figs. 9(b)-9(d). It is observable that the array-facing edges of the targets were recovered by all three methods, however, unlike in the simulations the object body could not be resolved because no light should have propagated through it. Despite of that, the outcomes by the three methods were different. For the 0 and $5 \mathrm{~mm}$ cases, the array-facing edge of the object was identified by the baseline method and the referencecompensation method at positions more proximal to the set positions, but not as sharp as by the GSD method. For $10 \mathrm{~mm}$ cases, artifacts were noticeable in all three methods, but the distortion to the object by the localized artifacts was the least in the GSD method.

\section{V.D. Experimental results for two-object cases}

The reconstruction results given in Figs. 10(b)-10(e) are similar to those in Fig. 8, except that for the $10 \mathrm{~mm}$ case the baseline method was able to resolve the two targets, but the depth was not recovered correctly. The GSD method slightly outperforms the other two methods in terms of resolving the location, dimension, and the optical properties of the two azimuthally separated targets.

\section{V.E. Simulation results of the simple three-dimensional imaging geometry}

Figure 11(c) displays the images reconstructed by using the baseline and the GSD approaches. By the baseline method the objects at different depths were recovered at depths closer 


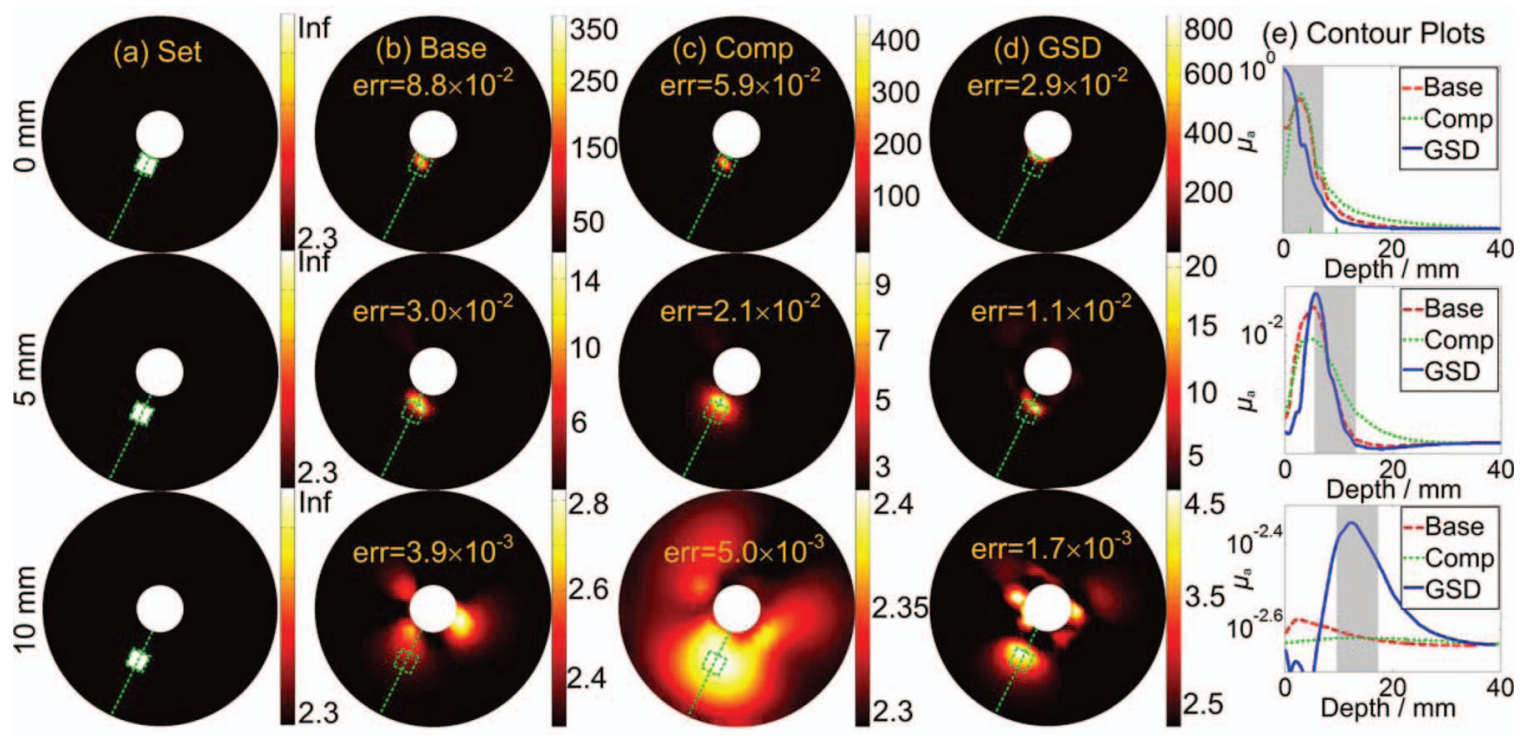

FIG. 9. Experimental results for single-object cases [unit used for columns (a)-(d): $10^{-3} \mathrm{~mm}^{-1}$, unit used for column (e): $\mathrm{mm}^{-1}$ ]. Column (a) set values; column (b) reconstruction based on the baseline method; column (c) reconstruction based on the reference-compensation method; column (d) reconstruction based on the GSD method; column (e) contour plots along the dotted lines marked in columns (b)-(d). The shaded areas in column (e) indicate the object locations corresponding to those marked in column (a). The "err"s in subfigures of columns (b)-(d) represent the standard deviation of the reconstruction errors averaged by the number of components in the objective function. Artifacts appeared for the object at $10 \mathrm{~mm}$-depth by all three methods. Column (d) has the best overall estimation of object location and domain optical properties.

to the optical array than the true values. At the depth of 30 $\mathrm{mm}$, the object was overwhelmed by the artifacts appearing close to the optical array when recovered by using the baseline method, in comparison, the depths and sizes of the object recovered by the GSD method were closer to the true values.

\section{DISCUSSION}

The layered DCA (Ref. 2) method was not directly implemented to the comparisons in this study, as a set of trials detailed in Appendix A indicated that the layered DCA method in its original form as in Ref. 2 was ineffective in

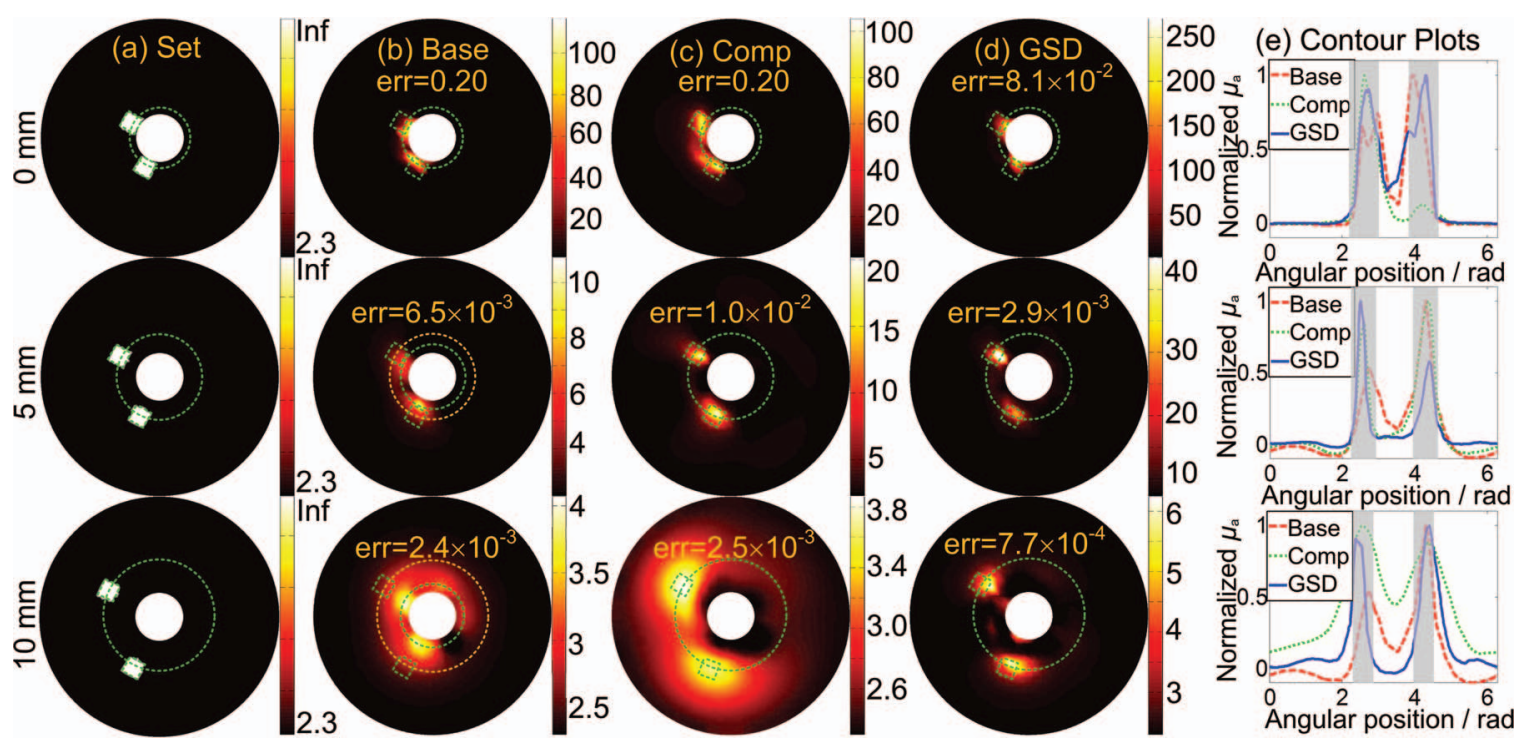

FIG. 10. Experimental results for dual-object cases [unit used for columns (a)-(d): $10^{-3} \mathrm{~mm}^{-1}$ ]. Column (a) set values; column (b) reconstruction based on the baseline; column (c) reconstruction based on the reference-compensation method; column (d) reconstruction based on the GSD method; column (e) normalized contour plots along the dotted loops marked in columns (b)-(d). The shaded areas in column (e) indicate the object locations corresponding to those marked in column (a). Note that although the widths of the object remain constant for all three depths, the radial expansion represented by the shaded areas in column (d) decreases with respect to imaging depth. The gold circles in column (d) indicate the object locations corresponding to those marked in column (a). The "err"s in subfigures of columns (b)-(d) represent the standard deviation of the reconstruction errors averaged by the number of components in the objective function. Column (d) has the best overall resolution of the two objects and the most accurate estimation of domain optical properties with minimal level of artifacts. 

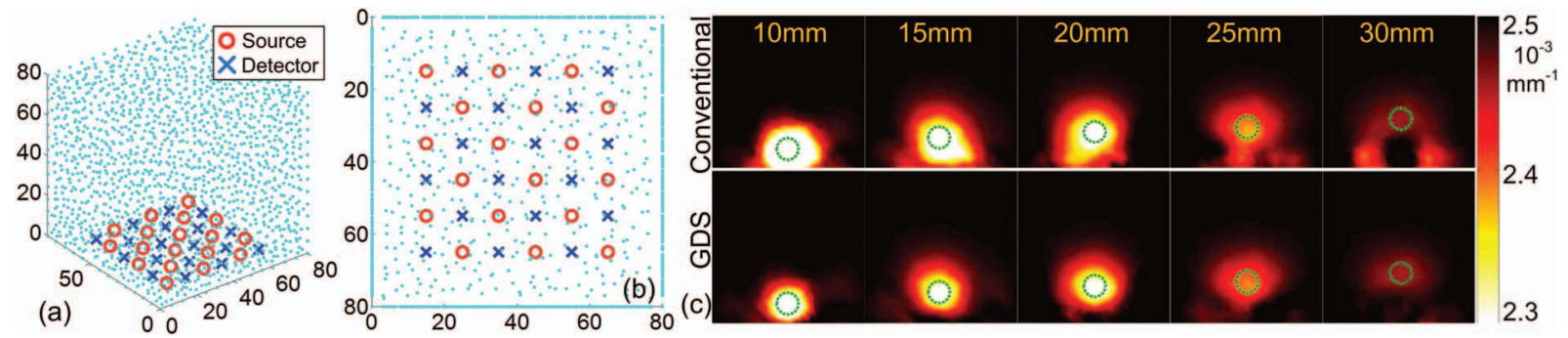

FIG. 11. Simulation results on recovering a single object in a planar reflectance imaging geometry. (a) 3D view of the simulating volume; (b) geometry of the optical array; (c) results of reconstruction based on the baseline and GSD methods. The objects are better resolved by GSD method.

the studied circular-array outward-imaging geometry. We argue that more robust suppression of the significant sensitivity decay of the studied geometry by using DCA method is feasible, should the resolution of the depth-layers be made comparable to or even finer than the radial resolution of the finite elements. We thus developed a piece-wise ${ }^{8}$ DCA-based reference-compensation method as detailed in Appendix B, and since this enhanced DCA method better balances between object localization and artifact suppression it is adopted as the reference-compensation method to facilitate the evaluation of GSD method.

The reference-compensation method localized the objects but generally underestimated the optical contrasts and overestimated the azimuthal FWHM of the objects, resulting in an ambiguous differentiation of two azimuthally separated objects. As to the baseline method that reconstructs using the native sensitivity distribution, it failed to resolve two objects at $10 \mathrm{~mm}$ depth with 5 folds of absorption contrast over the background [Fig. 8, row 3, column (b)]. However, in the corresponding experiment [Fig. 10, row 3, column (b)], the two black plastic objects placed at $10 \mathrm{~mm}$ depth were resolved by the baseline method. The better resolution of the two objects in the experiment than in the simulation was indeed the result of stronger signal change in the experiment than in the simulation due to the use of black materials for the objects in the experiments. The averaged errors of the reconstruction by the reference-compensation and baseline methods were nearly an order greater than those achieved by the GSD method. It is also observable that lesser artifacts arouse in the two target cases (Fig. 8 and Fig. 10) than in one target cases (Fig. 6 and Fig. 9) by the GSD-method. This may also have been related to the stronger signal change occurring to more sourcedetector pairs by two targets than that by one target, but it favorably indicates that the GSD method is robust in recovering multiple objects. It is also cautiously observed that the deeper the object is located, the less accurate the optical contrast is recovered, even for the GSD method. This implies that the effectiveness of how GSD performs, in terms of passive sensitivity compensation, diminishes as the measurement sensitivity deteriorates. More enhancement to the recovery of deeply embedded target by the GSD method is feasible, should the source power or signal to noise ratio become stronger.

One limitation of the experimental study regarding recovering two-objects is that the two objects were placed azimuthally at the same imaging depth, as the optode array was primarily designed for transrectal prostate cancer imaging, where the malignant tissues could be multifocal and usually occurring close to the optode array. Moreover, for the endoscopic imaging geometry in Fig. 3, because of the dimensions of the object tested, placing two objects of the shown sizes along the same radial direction, and not stacking them against each other for them to be reasonably resolved by the reconstruction, would need to place most of the distal object in a region beyond what could be measured by the experimental system, regardless of how the sensitivity variation would be managed. Using objects much smaller than those implemented should facilitate testing the GSD operations to radially separated objects in the shown geometry; however, the experimental system at the given low level of the source power would be much less sensitive to the smaller objects and the resulted poor signal-to-noise ratio would likely make any comparisons inconclusive.

The GSD method reduces the depth-variance of the resulted sensitivity at a cost of increasing the number of rows of the sensitivity matrix by (NOD-1)/2 folds, for source-sharing GSD operation. The increase of the number of rows of the sensitivity matrix, however, does not degrade the rank of the matrix to be inverted, since $\tilde{J}^{T} \tilde{J}$ has a $\mathrm{N} \times \mathrm{N}$ dimension as $J^{T} J$ does. The increase in the computation time, approximately $40 \%$ in this study, is solely caused by the [Diff] operation in Eq. (14) and the corresponding multiplication of matrices of more elements. A sensitivity distribution that is more depth-invariant than the GSD sensitivity distribution investigated in this study could be achievable by hierarchical pairing of the GSD-resulted sensitivity profiles, but the cost in computation and complexity would likely outweigh the benefits.

It is noted that the linear conversion from the $J$ to $\tilde{J}$ retains the full dynamic range of the measurements as is $\{\max (\Psi)-$ $\min (\Psi)\}$. The differentiated objective function in Eq. (15) can also be considered belonging to the scheme involving a surrogate objective function, ${ }^{22}$ as is demonstrated by Xu et al. ${ }^{23}$ for DOT image reconstruction using a spectral derivative method. The spectral derivative method is based on the assumption that the boundary modeling errors are multiplicative to system measurements in normal scale ${ }^{24}$ and can be modeled as additive in logarithm. The GSD method is similar to the spectral derivative methods in partially and passively cancelling out the modeling or system errors measured by two optodesharing source-detector-pairs. 
This study implemented the GSD operation in the LM scheme. It is known that the LM scheme is a special case of the Tikhonov regularization method, and the Tikhonov regularization itself is a special case of a generalized-least-square GLS approach. ${ }^{13,25}$ Because the operation on the $J$ by GSD method is linear, we expect that the GSD method is extendable to the Tikhonov and GLS minimization schemes. The GSD-operated update function in a Tikhonov minimization would become

$$
\delta \mu=\left(\tilde{J}^{T} \tilde{J}+\lambda I\right)^{-1} \times\left[\tilde{J}^{T} \tilde{\chi}-\lambda\left(\mu-\mu_{0}\right)\right]
$$

and the GSD-operated update function in a GLS minimization would become

$$
\begin{aligned}
\delta \mu= & {\left[\tilde{J}^{T} W_{\tilde{\chi}} \tilde{J}+W_{\mu-\mu_{0}}\right]^{-1} } \\
& \times\left[\tilde{J}^{T} W_{\tilde{\chi}} \tilde{\chi}-W_{\mu-\mu_{0}}\left(\mu-\mu_{0}\right)\right],
\end{aligned}
$$

where $W_{\tilde{\chi}}$ is the inverse of the covariance of the GSDoperated objective function $\tilde{\chi}$ and $W_{\mu-\mu_{0}}$ is the inverse of the covariance of the optical properties.

The effectiveness of the GSD method is apparently related to the extent of the spatial variations of the measurement sensitivity. The more uniform the native measurement sensitivity is with respect to the imaging depth, the less improvement the GSD method could impose to the object localization in the depth. On the other hand, the effectiveness of the GSD operation can be evaluated from Eq. (13) that it depends upon the geometric symmetry or antisymmetry of all source-detectorpairs. The circular-array imaging geometry as the one shown in Fig. 3 renders the geometric symmetry of $\left\langle S_{i}, D_{j}\right\rangle$ versus $\left\langle S_{i}, D_{9-j}\right\rangle$ for $i=\{1,5\}, j=\{1: 8\}$; and the geometric antisymmetry of the pairing of $\left[\left\langle S_{i}, D_{j}\right\rangle-\left\langle S_{i}, D_{9-j}\right\rangle\right]$ versus the pairing of $\left[\left\langle S_{10-i}, D_{j}\right\rangle-\left\langle S_{10-i}, D_{9-j}\right\rangle\right]$ for $i=\{2: 4\} ; j=\{1: 4\}$. These symmetries indicate that the spatial variation of the sensitivity of measurements introduced by an individual sourcedetector-pair $\left\langle S_{i}, D_{j}\right\rangle$ may be cancelled or partially compensated by other source-detector-pair. The circular-imaging geometry with evenly interspersed source and detector renders complete rotational symmetry in either directions of the circumference; therefore, it would be the geometry for which the application of the GSD operation would be the most effective. The simple three-dimensional geometry simulated in Secs. IV.D and IV.E has a lateral symmetry with respect to the two middle-sectioning planes, but the symmetry is not complete for all source-detector-pairs. Therefore, the GSD method to this geometry is not as effective as is to the circular-array outward-imaging geometry. The simulations performed on the simple three-dimensional geometry, nonetheless, demonstrate that GSD operation is generally applicable, and as such we anticipate that DOT reconstructions that relies upon more complex data, such as frequency domain, time domain, multispectral, or fluorescence measurements, may become the future subjects for implementing GSD-operation, should the correct localization of an object depth without a spatial prior become a concern.

\section{CONCLUSION}

This study demonstrated an alternative DOT image reconstruction approach that optimizes the data-model fit based on the paired measurements corresponding to two source-sharing source-detector-pairs, in comparison to the conventional DOT image reconstruction approach that optimizes the data-model fit based on the measurements corresponding to individual source-detector-pairs. This alternative method, conceptually called the GSD method, effectively and passively suppresses the spatial variance of the detection sensitivity with respect to imaging depth by taking advantage of the relative changes of the measurements between two optode-sharing sourcedetector-pairs. It is demonstrated that this GSD approach improves the object depth-localization for DOT in a circulararray outward-imaging geometry that is subjected to strong variation of the native measurement sensitivity with respect to the imaging depth. This GSD operation pairs the native sensitivity profiles corresponding to two source-sharing sourcedetector-pairs and effectively evens the responses of the updating function with respect to the imaging depth. Simulated and experimental studies based on $\mathrm{CW}$ measurements are used to evaluate the image reconstructions based on the GSDmethod versus that on two other methods: one is the baseline method using the native sensitivity that does not involve a depth-compensating scheme, and the other is a referencecompensation approach that involves active depth-dependent compensating scheme. The GSD method is shown generally outperforming the other two methods in terms of localizing the depth of single object, resolving two azimuthally separated objects, as well as estimating the optical property of single object or azimuthally separated dual objects. The GSD method, however, is more computationally demanding due to increasing of the matrix elements as well as involving more matrix multiplications, and the effectiveness of it is associated with the symmetry of the optode-array.

\section{ACKNOWLEGMENT}

This work is a result of a U.S. Army Medical Research Acquisition Activity Grant No. W81XWH-10-1-0836.

\section{APPENDIX A: LAYERED DCA METHOD APPLIED TO THE CIRCULAR-ARRAY OUTWARD-IMAGING GEOMETRY}

This section tests the DCA method in the form originally presented in Ref. 2 to the studied circular-array outwardimaging geometry. The DCA method actually introduces a compensation matrix similar to the weight matrix in the GLS method. ${ }^{13}$ The structure of the compensation matrix $M$ is represented using the notations consistent with this study as

$$
\begin{aligned}
M= & \left\{\operatorname { d i a g } \left[\operatorname{MSV}\left(J_{l}\right), \operatorname{MSV}\left(J_{l-1}\right), \ldots,\right.\right. \\
& \left.\left.\operatorname{MSV}\left(J_{2}\right), \operatorname{MSV}\left(J_{1}\right)\right]\right\}^{\gamma},
\end{aligned}
$$

where MSV stands for the maximum singular values, $J_{l}$ is the sensitivity terms in the layer $l$, and $\gamma$ is an exponential factor to be empirically optimized for a specific geometry. The 

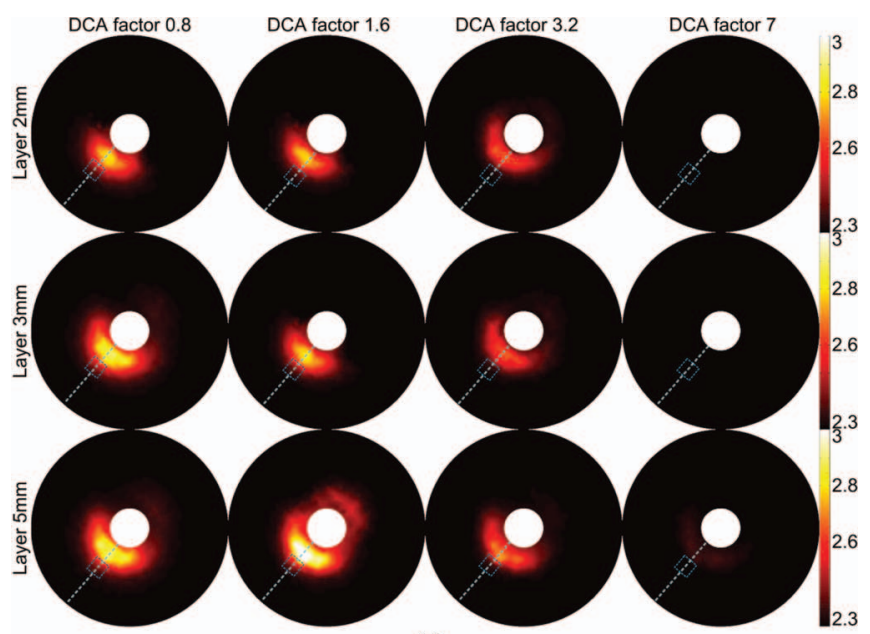

(a)

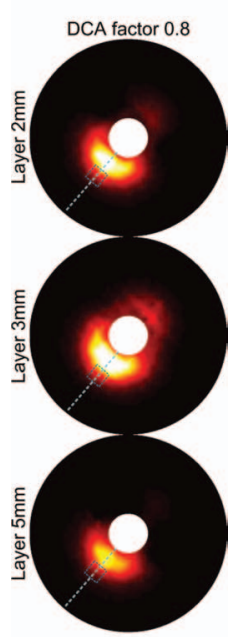

DCA factor 1.

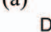

DCA factor 3.2

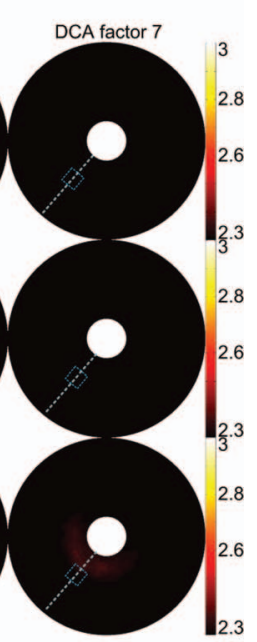

(b)
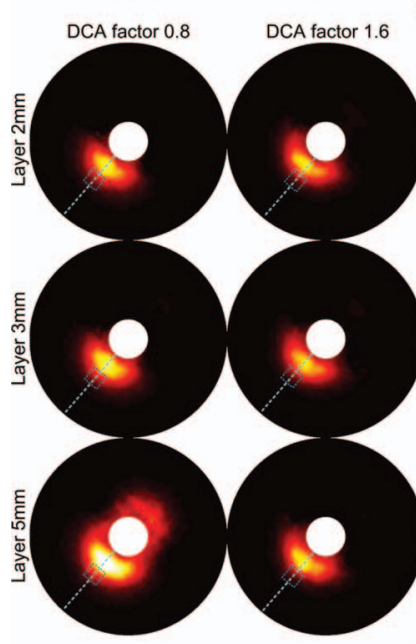

DCA factor 3.2
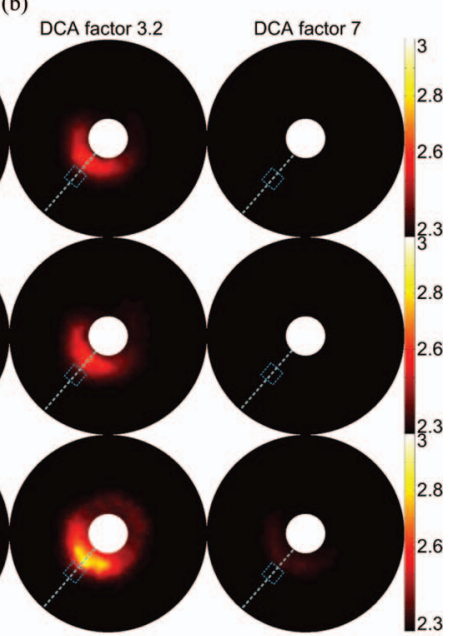

(c)

FIG. 12. Investigation on the performance of DCA method in the circulararray outward-imaging geometry (units $10^{-3} \mathrm{~mm}^{-1}$ ). The object contour and location are marked by blue dotted lines. Simulation setup in Sec. III.B for single object was used. (a)-(c) Simulation results produced with regularization factor value of 50,100, and 200, respectively. The DCA method in its original form as in Ref. 2 was ineffective in the studied circular-array outward-imaging geometry.

gradient-based iterative solver as that shown in Ref. 2 is implemented in the inverse problem by using the compensation sensitivity matrix $M J$. Note that $M$ is a square matrix and can be categorized to the GLS method.

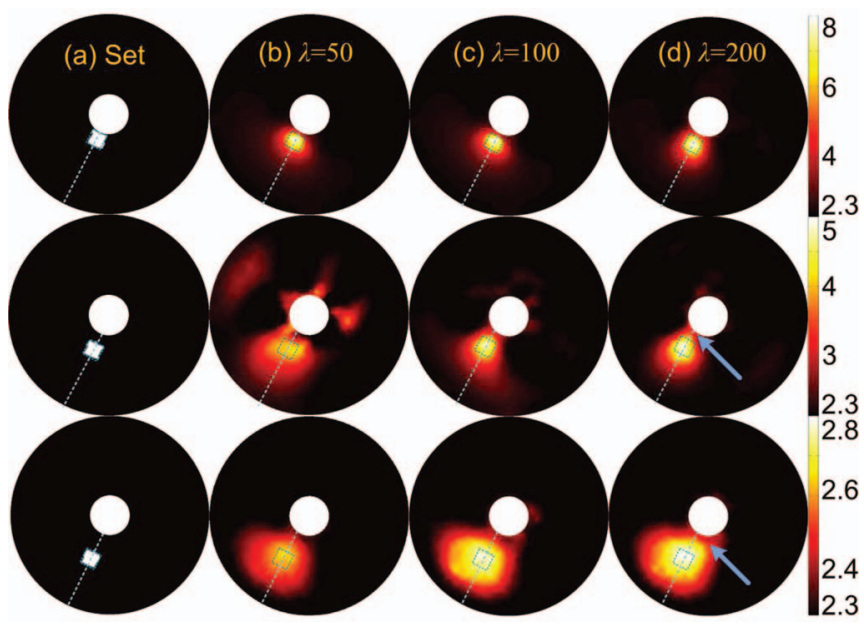

FIG. 13. Performance of the reference compensation method with varied regularization factors (units $10^{-3} \mathrm{~mm}^{-1}$ ). The object contour and location are marked by dotted lines. When the object was close to the probe surface, reconstruction results are not significantly affected by the regularization factor. At deeper locations, either more artifacts appear or the object contrast is more severely underestimated in the column of $\lambda=50$. The columns of $\lambda=$ 100 and $\lambda=200$ are only slightly different; however, the target size seems to be slightly overestimated by $\lambda=200$ than by $\lambda=100$. The arrows indicate the overestimations of the optical properties of the medium region between the object and the applicator.

Three parameters were tested. The first parameter was the thickness of the layer that was chosen to be 2,3 , and $5 \mathrm{~mm}$, receptively, based on the $1 \mathrm{~mm}$ radial resolution of the mesh in this simulation and the $5 \mathrm{~mm}$ step of changing the object depth in Sec. IV. The second parameter was the exponential factor. Niu et al. ${ }^{2}$ tested that $\gamma=1.6$ was optimal for the planar reflectance imaging geometry specific to their study. In the circular-array outward imaging geometry of this study a series of $\gamma$ values between 0 and 10 were tested, yet only the reconstruction results produced with $\gamma$ values of $\{0.8,1.6$, $3.2,7\}$ were presented in this section. The $\gamma$ values were chosen greater than those used in Ref. 2, which was found necessary to counteract the more severe decay of the sensitivity with respect to imaging depth. The third parameter was the regularization factor $\lambda$ that is critical to the convergence of the inverse problem, and the $\lambda$ values of $\{50,100,200\}$ were tested.

The test simulation shown in Fig. 12 is conducted for single cubic object identical to the one in Fig. 6 and its proximaledge located at a depth of $10 \mathrm{~mm}$. The objects in all cases were recovered at depths much closer to the probe surface than the true values, indicating the inadequacy of the DCA method in the tested or the originally presented form for the studied geometry. Implementing the DCA method in a piece-wise exponential weighting scheme is found to produce better results, as shown in Appendix B and Sec. V.

\section{APPENDIX B: DETERMINATION OF THE INITIAL REGULARIZATION VALUE USED FOR STEP-WISE ADJUSTMENT}

Because of the poor depth-localization of the baseline reconstruction in the studied geometry, the $\lambda$ value to be 
step-wisely adjusted ${ }^{13}$ was actually determined based on the reference compensation methods of Eq. (17), and the same stepwise-adjusting of the $\lambda$ value was applied to the baseline method. A wide range of $\lambda$ values were examined, for the single object case identical to that in Sec.IV.A, and those shown in Fig. 13 were the results of using three representative $\lambda$ values of $\{50,100,200\}$. It is clear that in the column of $\lambda$ $=50$ either there are more artifacts or the object contrast is more severely underestimated. The columns of $\lambda=100$ and $\lambda=200$ are not significantly different; however, $\lambda=200$ slightly overestimates the target size than $\lambda=100$ does; therefore, a $\lambda$ value of 100 is chosen.

a) Author to whom correspondence should be addressed. Electronic mail: daqing.piao@okstate.edu

${ }^{1}$ M. Huang and Q. Zhu, "Dual-mesh optical tomography reconstruction method with a depth correction that uses a priori ultrasound information," Appl. Opt. 43(8), 1654-1662 (2004).

${ }^{2}$ H. Niu, F. Tian, Z.-J. Lin, and H. Liu, "Development of a compensation algorithm for accurate depth localization in diffuse optical tomography," Opt. Lett. 35(3), 429-431 (2010).

${ }^{3}$ G. Xu, D. Piao, C. H. Musgrove, C. F. Bunting, and H. Dehghani, “Transrectal ultrasound-coupled near-infrared optical tomography of the prostate, Part I: Simulation,” Opt. Express 16(22), 17484-17504 (2008).

${ }^{4}$ Q. Zhao, L. Ji, and T. Jiang, "Improving depth resolution of diffuse optical tomography with a layer-based sigmoid adjustment method," Opt. Express 15(7), 4018-4029 (2007).

${ }^{5}$ S. R. Arridge, "Optical tomography in medical imaging," Inverse Probl. 15(2), R41-R93 (1999).

${ }^{6}$ D. S. Kepshire, S. C. Davis, H. Dehghani, K. D. Paulsen, and B. W. Pogue, "Subsurface diffuse optical tomography can localize absorber and fluorescent objects but recovered image sensitivity is nonlinear with depth," Appl. Opt. 46(10), 1669-1678 (2007).

${ }^{7}$ F. Tian, G. Alexandrakis, and H. Liu, "Optimization of probe geometry for diffuse optical brain imaging based on measurement density and distribution," Appl. Opt. 48(13), 2496-2504 (2009).

${ }^{8}$ B. W. Pogue, T. O. McBride, J. Prewitt, U. L. Österberg, and K. D. Paulsen, "Spatially variant regularization improves diffuse optical tomography," Appl. Opt. 38(13), 2950-2961 (1999).

${ }^{9}$ D. Piao, H. Xie, C. Musgrove, C. F. Bunting, W. Zhang, G. Zhang, E. B. Domnick-Davidsion, K. E. Bartels, G. R. Holyoak, S. N. Vemulapalli, H. Dehghani, and B. W. Pogue, Near-Infrared Optical Tomography: Endoscopic Imaging Approach, Proc. SPIE 6431, Multimodal Biomedical Imaging II, 643103 (2007).

${ }^{10}$ J. P. Culver, A. M. Siegel, J. J. Stott, and D. A. Boas, "Volumetric diffuse optical tomography of brain activity," Opt. Lett. 28(21), 2061-2063 (2003)
${ }^{11}$ A. Zhang, D. Piao, C. F. Bunting, and B. W. Pogue, "Photon diffusion in a homogeneous medium bounded externally or internally by an infinitely long circular cylindrical applicator. I. Steady-state theory," J. Opt. Soc. Am. A 27(3), 648-662 (2010).

${ }^{12}$ D. W. Marquardt, "An algorithm for least-squares estimation of nonlinear parameters,” J. Soc. Ind. Appl. Math. 11(2), 431-441 (1963).

${ }^{13}$ P. K. Yalavarthy, B. W. Pogue, H. Dehghani, and K. D. Paulsen, "Weightmatrix structured regularization provides optimal generalized least-squares estimate in diffuse optical tomography," Med. Phys. 34(6), 2085-2098 (2007).

${ }^{14}$ H. Dehghani, M. E. Eames, P. K. Yalavarthy, S. C. Davis, S. Srinivasan, C. M. Carpenter, B. W. Pogue, and K. D. Paulsen, "Near infrared optical tomography using NIRFAST: Algorithm for numerical model and image reconstruction," Commun. Numer. Methods Eng. 25(6), 711-732 (2009).

${ }^{15}$ A. Corlu, R. Choe, T. Durduran, K. Lee, M. Schweiger, S. R. Arridge, E. M. C. Hillman, and A. G. Yodh, "Diffuse optical tomography with spectral constraints and wavelength optimization," Appl. Opt. 44(11), 2082-2093 (2005).

${ }^{16}$ H. Bastian, "On uniqueness in diffuse optical tomography," Inverse Probl. 25(5), 055010 (14pp) (2009).

${ }^{17}$ Y. Xu, X. Gu, T. Khan, and H. Jiang, "Absorption and scattering images of heterogeneous scattering media can be simultaneously reconstructed by use of dc data," Appl. Opt. 41(25), 5427-5437 (2002).

${ }^{18}$ G. Xu, D. Piao, C. F. Bunting, and H. Dehghani, "Direct-current-based image reconstruction versus direct-current included or excluded frequencydomain reconstruction in diffuse optical tomography," Appl. Opt. 49(16), 3059-3070 (2010).

${ }^{19}$ H. Niu, Z.-J. Lin, F. Tian, S. Dhamne, and H. Liu, "Comprehensive investigation of three-dimensional diffuse optical tomography with depth compensation algorithm," J. Biomed. Opt. 15(4), 046005-046009 (2010).

${ }^{20}$ V. Ntziachristos, A. G. Yodh, M. Schnall, and B. Chance, "Concurrent MRI and diffuse optical tomography of breast after indocyanine green enhancement," Proc. Natl. Acad. Sci. U.S.A. 97(6), 2767-2772 (2000).

${ }^{21}$ G. Xu and D. Piao, Feasibility of Rapid Near-Infrared Diffuse Optical Tomography by Swept-Spectral-Encoded Sequential Light Delivery, Proc. SPIE 7896, Optical Tomography and Spectroscopy of Tissue IX, 78961W (2011).

${ }^{22}$ K. Lange, D. R. Hunter, and Y. Ilsoon, "Optimization transfer using surrogate objective functions," J. Comput. Graph. Stat. 9(1), 1-20 (2000).

${ }^{23}$ H. Xu, B. W. Pogue, R. Springett, and H. Dehghani, "Spectral derivative based image reconstruction provides inherent insensitivity to coupling and geometric errors," Opt. Lett. 30(21), 2912-2914 (2005).

${ }^{24}$ M. Schweiger, I. Nissilä, D. A. Boas, and S. R. Arridge, "Image reconstruction in optical tomography in the presence of coupling errors," Appl. Opt. 46(14), 2743-2756 (2007).

${ }^{25}$ P. K. Yalavarthy, D. R. Lynch, B. W. Pogue, H. Dehghani, and K. D. Paulsen, "Implementation of a computationally efficient least-squares algorithm for highly under-determined three-dimensional diffuse optical tomography problems," Med. Phys. 35(5), 1682-1697 (2008). 